\title{
Lie description of higher obstructions to deforming submanifolds
}

\author{
MARCO MANETTI
}

\begin{abstract}
To every morphism $\chi: L \rightarrow M$ of differential graded Lie algebras we associate a functors of artin rings $\operatorname{Def}_{\chi}$ whose tangent and obstruction spaces are respectively the first and second cohomology group of the suspension of the mapping cone of $\chi$.

Such construction applies to Hilbert and Brill-Noether functors and allow to prove with ease that every higher obstruction to deforming a smooth submanifold of a Kähler manifold is annihilated by the semiregularity map.
\end{abstract}

Mathematics Subject Classification (2000): 13D10 (primary); 14D15 (secondary).

\section{Introduction}

This paper is devoted, as its ancestors [26,27], to develop algebraic tools that are useful to handle deformation problems over a field of characteristic 0 . The philosophy underlying this project, due to Deligne, Drinfeld and Kontsevich, is that every "reasonable" deformation problem is the truncation of an extended deformation problem which is governed by a differential graded Lie algebra (DGLA) and every morphism of deformation theories is induced by an $L_{\infty}$-morphism.

Usually, the formal deformations of an algebro-geometric structure are described by a functor of Artin rings [1,31]. The link between differential graded Lie algebras and functors of Artin rings is given by the Maurer-Cartan equation. More precisely, to every differential graded Lie algebra $L$ it is associated the functor $[13,14,24,25]$

$$
\begin{gathered}
\operatorname{Def}_{L}:\{\text { local Artinian rings }\} \rightarrow\{\text { Sets }\} \\
\operatorname{Def}_{L}(A)=\frac{\left\{x \in L^{1} \otimes \mathfrak{m}_{A} \mid d x+[x, x] / 2=0\right\}}{\text { gauge equivalence }}
\end{gathered}
$$

where $\mathfrak{m}_{A}$ is the maximal ideal of $A$. The functor $\operatorname{Def}_{L}$ depends only on the quasiisomorphism class of $L$. In practice, the advantage of this approach concerns especially the study of higher obstructions. Classically these obstructions were studied

Received April 18, 2007; accepted in revised form November 14, 2007. 
using Massey products (see e.g. [9, 29]) but, as well explained in [32], "Massey product structures can be very helpful, though they are in general described in a form that is unsatisfactory."

In most cases, the replacing of the Massey product structure with the appropriate DGLA structure allow one to prove new results and easier proofs of old results. On the other side it is not always easy to determine the right DGLA governing a deformation problem.

The first goal of this paper is to develop some algebraic tools that are useful to relate differential graded Lie algebras and semitrivial deformations. The embedded deformations of a submanifold is the most classical example of semitrivial deformation problem. Roughly speaking, we have a manifold $X$ and a submanifold $Z \subset X$. The idea is to consider the embedded deformation of $Z$ in $X$ as the deformations of the inclusion map $Z \hookrightarrow X$ inducing a trivial deformation of $X$. One other classical example is the Brill-Noether functor, i.e. deformations of a bundle inducing trivial deformation of its cohomology. The key point is the definition of a functor Def $_{\chi}$ associated to a morphism $\chi: L \rightarrow M$ of differential graded Lie algebras and the verification that this functor has the same formal properties as $\operatorname{Def}_{L}$; in particular the implicit function theorem (Theorem 2.1) still holds. Then, using the trick of path-objects we see that there exists a new DGLA $H$ such that $\operatorname{Def}_{\chi}=\operatorname{Def}_{H}$. This construction allows for example to determine a differential graded Lie algebra that governs embedded deformations and avoid the use, as in [5], of higher order differential operators. Our proof of implicit function theorem is based on the the theory of extended deformation functors (EDF), introduced in [26]. As a further motivation for the use of EDFs instead of the classical approach [13], we have that the extended deformation functors are good objects for studying the "local theory" in derived algebraic geometry: every EDF can be considered as a concrete representative for a quasiisomorphism class of differential graded Lie algebra and every property of a DGLA which is invariant under quasiisomorphism can be recovered from the associated extended deformation functor.

Recently, D. Iacono [18] has generalized the construction of this paper to a pair of morphisms of DGLA $\chi_{i}: L_{i} \rightarrow M, i=1,2$; as a consequence she find the DGLA governing deformations of holomorphic maps. On the other side, in the paper [11], we have interpreted the functor $\operatorname{Def}_{\chi}$ as the deformation functor of a canonical $L_{\infty}$ structure on the mapping cone of $\chi$.

The second goal of this paper is to check the utility of this approach on a series of concrete examples; in particular we are able to prove the following result (Theorem 8.1).

Theorem 0.1. Let $Z$ be a smooth closed submanifold of a compact Kähler manifold $X$ and let $\omega$ be a closed differential $(p, q)$-form on $X$ such that $\omega_{\mid Z}=0$. Then the obstructions of embedded deformations of $Z$ inside $X$ are contained in the kernel of the contraction map

$$
\lrcorner \omega: H^{1}\left(Z, N_{Z \mid X}\right) \rightarrow H^{q+1}\left(Z, \Omega_{Z}^{p-1}\right) .
$$


It has to be observed that a very similar result has been recently proved by $\mathrm{H}$. Clemens in [6, Theorem 12.1]. As applications of Theorem 0.1 we get the vanishing of all higher obstructions to embedded deformations under Bloch's semiregularity map (Corollary 8.2) and the unobstructedness of Lagrangian submanifolds of a holomorphic symplectic variety (Corollary 8.5).

The basic difference of our result with respect to the similar ones in existing literature is that our theorem works for every obstruction, while the others based on Hodge theory [3,30] or cotangent complex [4] are valid only for obstructions arising from small extensions

$$
0 \longrightarrow \mathrm{J} \stackrel{f}{\longrightarrow} A \longrightarrow B \longrightarrow 0
$$

of Artin rings where the differential $d f: J \rightarrow \Omega_{A} \otimes_{A} B$ is injective. Although this doesn't make any difference about the possible unobstructedness of the Hilbert scheme and for dimension bounds, it is a substantial improvement when we are interested to virtual fundamental classes and obstruction theories in the sense of Behrend-Fantechi [2].

Acknowledgements. I'm indebted with Domenico Fiorenza for several and useful discussions on these topics. I also thank Donatella Iacono for having carefully read the preliminary versions of this paper. Thanks also to the referees for useful comments and suggestions for future developments.

\section{General notation}

We always work over the field $\mathbb{C}$, although most of the results of algebraic nature are valid over an arbitrary field of characteristic 0 . Unless otherwise specified the symbol $\otimes$ denotes the tensor product over $\mathbb{C}$.

The term DGLA means differential $\mathbb{Z}$-graded Lie algebras, while dg-algebra means a differential $\mathbb{Z}$-graded, graded-commutative and associative algebra.

Unless otherwise specified, every complex manifold is assumed compact and connected. For every complex manifold $X$ we denote by:

- $T_{X}$ the holomorphic tangent bundle on $X$.

- $\mathcal{A}_{X}^{p, q}$ the sheaf of differentiable $(p, q)$-forms of $X$. More generally if $E$ is a holomorphic vector bundle on $X$ we denote by $\mathcal{A}_{X}^{p, q}(E)$ the sheaf of differentiable $(p, q)$-forms of $X$ with values in $E$ and by $A_{X}^{p, q}(E)=\Gamma\left(X, \mathcal{A}_{X}^{p, q}(E)\right)$ the space of its global sections.

- For every submanifold $Z \subset X$, we denote by $N_{Z \mid X}$ the normal bundle of $Z$ in $X$. 


\section{Background}

Let Set be the category of sets and Art the category of local Artinian $\mathbb{C}$-algebras $(A, \mathfrak{m})$ with residue field $A / \mathfrak{m}=\mathbb{C}$. Following [31], by a functor of Artin rings we intend a covariant functor $\mathcal{F}:$ Art $\rightarrow$ Set such that $\mathcal{F}(\mathbb{C})=\{$ one element $\}$. By the term Schlessinger's condition we mean one of the four conditions $\left(\mathrm{H}_{1}\right), \ldots,\left(\mathrm{H}_{4}\right)$ described in [31, Theorem 2.1]. Given a functor $\mathcal{F}$ as above and morphisms $A \stackrel{\alpha}{\longrightarrow} C$, $B \stackrel{\beta}{\longrightarrow} C$ in Art, let $\eta$ be the natural map

$$
\eta: \mathcal{F}\left(A \times_{C} B\right) \rightarrow F(A) \times{ }_{F(C)} F(B) .
$$

Then the Schlessinger's conditions are:

$\mathrm{H}_{1} \eta$ is surjective if $\alpha$ is surjective.

$\mathrm{H}_{2} \eta$ is bijective if $A=\mathbb{C}[t] /\left(t^{2}\right), C=\mathbb{C}$.

$\mathrm{H}_{3} \quad \mathrm{H}_{1}$ and $\mathrm{H}_{2}$ hold and $\mathcal{F}\left(\mathbb{C}[t] /\left(t^{2}\right)\right)$ is finite dimensional as $\mathbb{C}$-vector space.

$\mathrm{H}_{4} \eta$ is bijective if $A=B, \alpha=\beta$ and $\operatorname{ker} \alpha=\operatorname{ker} \beta$ is annihilated by the maximal ideal.

Functors of Artin rings are used to describe infinitesimal deformations of algebrogeometric structures.

Example 1.1. Let $X$ be a complex manifold and let $Z \subset X$ be an analytic subvariety defined by a sheaf of ideals $\mathcal{I} \subset \mathcal{O}_{X}$. The infinitesimal embedded deformations of $Z$ in $X$ are described by the Hilbert functor $\operatorname{Hilb}_{X}^{Z}:$ Art $\rightarrow$ Set,

$\operatorname{Hilb}_{X}^{Z}(A)=\left\{\right.$ ideal sheaves $\mathcal{I}_{A} \subset \mathcal{O}_{X} \otimes_{\mathbb{C}} A$, flat over $A$ such that $\left.\mathcal{I}_{A} \otimes_{A} \mathbb{C}=\mathcal{I}\right\}$.

Let $K=\left(\oplus K^{i}, d,[],\right)$ be a differential graded Lie algebra; given $(A, \mathfrak{m}) \in$ Art, the set of Maurer-Cartan elements with coefficients in $A$ is by definition

$$
\operatorname{MC}_{K}(A)=\left\{x \in K^{1} \otimes \mathfrak{m} \mid d x+\frac{1}{2}[x, x]=0\right\},
$$

where the DGLA structure on $K \otimes \mathfrak{m}$ is given by the natural extension of the differential $d$ and the bracket [, ] defined as

$$
d(v \otimes a)=d(v) \otimes a, \quad[v \otimes a, w \otimes b]=[v, w] \otimes a b .
$$

Since $K^{0} \otimes \mathfrak{m}$ is a nilpotent Lie algebra, its exponential group $\exp \left(K^{0} \otimes \mathfrak{m}\right)$ can be defined as the set $\left\{e^{a} \mid a \in K^{0} \otimes \mathfrak{m}\right\}$; we have $e^{a} e^{b}=e^{a \bullet b}$, where $\bullet$ is the Baker-Campbell-Hausdorff product (see e.g. [19,28]).

The gauge action $\exp \left(K^{0} \otimes \mathfrak{m}\right) \times \mathrm{MC}_{K}(A) \rightarrow \mathrm{MC}_{K}(A)$ is given explicitly by the formula

$$
e^{a} * w=w+\sum_{n \geq 0} \frac{[a,-]^{n}}{(n+1) !}([a, w]-d a)
$$


Remark 1.2. The vector space $d K^{-1}$ is a Lie subalgebra of $K^{0}$ and $\exp \left(d K^{-1} \otimes \mathfrak{m}\right)$ is contained in the stabilizer of 0 . More generally, for every $x \in \operatorname{MC}_{K}(A)$ the subspace $(d+[x,-]) K^{-1} \otimes \mathfrak{m}$ is a Lie subalgebra of $K^{0} \otimes \mathfrak{m}$ and the group

$$
S_{x}(A)=\left\{e^{[x, h]+d h} \mid h \in K^{-1} \otimes \mathfrak{m}\right\}
$$

is contained in the stabilizer of $x$. It is easy to verify that for $a \in K^{0} \otimes \mathfrak{m}$ we have $e^{a} S_{x}(A) e^{-a}=S_{y}(A)$, where $y=e^{a} * x$. We shall call the group $S_{x}(A)$ the irrelevant stabilizer of $x$.

The functor $\operatorname{Def}_{K}:$ Art $\rightarrow$ Set is defined as the quotient of Maurer-Cartan by the gauge action,

$$
\operatorname{Def}_{K}(A)=\frac{\mathrm{MC}_{K}(A)}{\exp \left(K^{0} \otimes \mathfrak{m}\right)} .
$$

The functor Def ${ }_{K}$ satisfies the Schlessinger's conditions $\left(\mathrm{H}_{1}\right),\left(\mathrm{H}_{2}\right)($ see $[10,31])$ and its tangent space $\operatorname{Def}_{K}\left(\mathbb{C}[\epsilon] /\left(\epsilon^{2}\right)\right)$ is naturally isomorphic to $H^{1}(K)$. We point out for later use that, if $H^{1}(K)=0$, then the functor $\operatorname{Def}_{K}$ is trivial and then for every $x \in \mathrm{MC}_{K}(A)$ there exists $a \in K^{0} \otimes \mathfrak{m}$ such that $x=e^{a} * 0$. If $H^{1}(K)$ is finite dimensional and $H^{0}(K)=0$ then $\operatorname{Def}_{K}$ is prorepresentable.

Definition 1.3. We say that a functor of Artin rings $\mathcal{F}:$ Art $\rightarrow$ Set is governed by a differential graded Lie algebra $K$ if $\mathcal{F}$ is isomorphic to $\operatorname{Def}_{K}$.

Example 1.4. Let $E$ be a holomorphic vector bundle on a complex manifold $X$, then the functor of infinitesimal deformations of $E$ is governed by the differential graded Lie algebra

$$
K=\oplus_{i \geq 0} K^{i}, \quad K^{i}=A_{X}^{0, i}(\operatorname{End}(E)),
$$

endowed with the Dolbeault differential and the natural bracket. More precisely if $e, g$ are local holomorphic sections of $\operatorname{End}(E)$ and $\phi, \psi$ differential forms we define $d(\phi e)=(\bar{\partial} \phi) e,[\phi e, \psi g]=\phi \wedge \psi[e, g]$. We refer to [12], [21, Chapter VII], [13, Section 9.4], [8, Page 238] for the proof that $\operatorname{Def}_{K}$ is isomorphic to the functor of infinitesimal deformations of $E$. Here we only note that, for every $(A, \mathfrak{m}) \in \operatorname{Art}$ and every $x \in \operatorname{MC}_{K}(A)$, the associated deformation of $E$ over $\operatorname{Spec}(A)$ is the bundle whose sheaf of holomorphic section is the kernel of

$$
\bar{\partial}+x: \mathcal{A}_{X}^{0,0}(E) \otimes A \rightarrow \mathcal{A}_{X}^{0,1}(E) \otimes A .
$$

\section{Mapping cone of DGLA morphisms}

Let $\chi: L \rightarrow M$ be a morphism of differential graded Lie algebras; by definition the mapping cone of $\chi$ is the complex (see e.g. [17]) $\operatorname{Cone}(\chi)$, where $\operatorname{Cone}(\chi)^{i}=$ $L^{i+1} \oplus M^{i}$ and the differential is given by the formula

$$
L^{i+1} \oplus M^{i} \ni(l, m) \mapsto(-d l,-\chi(l)+d m) \in L^{i+2} \oplus M^{i+1} .
$$


We are interested in the suspension of the mapping cone (SMC) of $\chi$; it is the differential graded vector space $\left(C_{\chi}, \delta\right)$, where $C_{\chi}^{i}=L^{i} \oplus M^{i-1}$ and the differential $\delta$ is defined as

$$
\delta(l, m)=(d l, \chi(l)-d m) .
$$

The projection $C_{\chi} \rightarrow L$ is a morphism of complexes and there exist boundary operators $H^{i}(M) \rightarrow H^{i+1}\left(C_{\chi}\right)$ giving a long exact sequence

$$
\cdots \rightarrow H^{i}\left(C_{\chi}\right) \rightarrow H^{i}(L) \stackrel{\chi}{\longrightarrow} H^{i}(M) \rightarrow H^{i+1}\left(C_{\chi}\right) \rightarrow \cdots
$$

In general, there does not exist any bracket on the suspended mapping cone making $C_{\chi}$ a DGLA and the projection $C_{\chi} \rightarrow L$ a Lie morphism. Nevertheless, there exists a natural notion of Maurer-Cartan equation and gauge action and we are able to define the associated deformation functor. For every local Artinian $\mathbb{C}$-algebra $(A, \mathfrak{m})$ we define

$$
\begin{aligned}
& \operatorname{MC}_{\chi}(A)=\left\{\left(x, e^{a}\right) \in\left(L^{1} \otimes \mathfrak{m}\right) \times \exp \left(M^{0} \otimes \mathfrak{m}\right) \mid d x+\frac{1}{2}[x, x]=0, e^{a} * \chi(x)=0\right\}, \\
& \operatorname{Def}_{\chi}(A)=\frac{\operatorname{MC}_{\chi}(A)}{\exp \left(L^{0} \otimes \mathfrak{m}\right) \times \exp \left(d M^{-1} \otimes \mathfrak{m}\right)},
\end{aligned}
$$

where the gauge action is given by the formula

$$
\left(e^{l}, e^{d m}\right) *\left(x, e^{a}\right)=\left(e^{l} * x, e^{d m} e^{a} e^{-\chi(l)}\right)=\left(e^{l} * x, e^{d m \bullet a \bullet(-\chi(l))}\right) .
$$

We now analyze the main properties of the functors $\operatorname{Def}_{\chi}$.

\section{Functoriality}

Every commutative diagram of differential graded Lie algebras

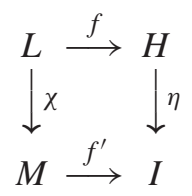

induces a natural transformation of functors $\operatorname{Def}_{\chi} \rightarrow \operatorname{Def}_{\eta}$ and a morphism of complexes of vector spaces $C_{\chi} \rightarrow C_{\eta}$.

Theorem 2.1 (Inverse function theorem). If the diagram (1.1) induces a quasiisomorphism $C_{\chi} \rightarrow C_{\eta}$, then the natural transformation $\operatorname{Def}_{\chi} \rightarrow \operatorname{Def}_{\eta}$ is an isomorphism. 
The proof is quite long and, for the clarity of exposition, it is postponed to Section 6.

If $K$ is the kernel of a morphism $\chi: L \rightarrow M$ of DGLA, then there exist natural transformations

$$
\operatorname{Def}_{K} \rightarrow \operatorname{Def}_{\chi} \stackrel{\pi}{\longrightarrow} \operatorname{Def}_{L}
$$

If $\chi$ is surjective, then $\operatorname{Def}_{K} \simeq \operatorname{Def}_{\chi}$; if $M$ is acyclic, then $\operatorname{Def}_{\chi} \simeq \operatorname{Def}_{L}$.

\section{Tangent space}

The tangent space of a functor $\mathcal{F}$ is by definition the space $\mathcal{F}(\mathbb{C}[\epsilon])$, where $\epsilon^{2}=0$. Therefore

$$
\begin{aligned}
\operatorname{MC}_{\chi}(\mathbb{C}[\epsilon]) & =\left\{\left(x, e^{a}\right) \in\left(L^{1} \otimes \mathbb{C} \epsilon\right) \times \exp \left(M^{0} \otimes \mathbb{C} \epsilon\right) \mid d x=0,\right. \\
\left.e^{a} * \chi(x)=\chi(x)-d a=0\right\} & \\
& \simeq\left\{(x, a) \in L^{1} \times M^{0} \mid d x=0, \quad \chi(x)-d a=0\right\}=\operatorname{ker}\left(\delta: C_{\chi}^{1} \rightarrow C_{\chi}^{2}\right) .
\end{aligned}
$$

Two elements $(x, a),(y, b) \in \operatorname{ker} \delta$ are gauge equivalent if and only if there exists $(c, z) \in L^{0} \times M^{-1}$ such that

$y=x-d c, \quad b=d z+a-\chi(c), \quad$ or equivalently $\quad(x, a)-(y, b)=\delta(c, z)$.

In conclusion the tangent space of $\operatorname{Def}_{\chi}$ is isomorphic to $H^{1}\left(C_{\chi}\right)$.

\section{Obstructions}

The obstruction space of $\operatorname{Def}_{\chi}$ is naturally contained in $H^{2}\left(C_{\chi}\right)$. Consider in fact a small extension in Art

$$
0 \longrightarrow \mathbb{C} \longrightarrow A \stackrel{\alpha}{\longrightarrow} B \longrightarrow 0
$$

and let $\left(x, e^{q}\right) \in \mathrm{MC}_{\chi}(B)$.

Since $\alpha$ is surjective there exists a pair $\left(y, e^{p}\right) \in L^{1} \otimes \mathfrak{m}_{A} \times \exp \left(M^{0} \otimes \mathfrak{m}_{A}\right)$ such that $\alpha(y)=x$ and $\alpha(p)=q$. Setting

$$
h=d y+\frac{1}{2}[y, y] \in L^{2} \otimes \mathbb{C}, \quad r=e^{p} * \chi(y) \in M^{1} \otimes \mathbb{C}
$$

we have $\delta(h, r)=0$. In fact,

$$
d h=\frac{1}{2} d[y, y]=[d y, y]=[h, y]-\frac{1}{2}[[y, y], y] .
$$


By Jacobi identity $[[y, y], y]=0$, while $[h, y]=0$ because the maximal ideal of $A$ annihilates the kernel of $\alpha$; therefore $d h=0$. Since $\chi(y)=e^{-p} * r=r+e^{-p} * 0$, we have

$$
\begin{aligned}
\chi(h) & =d\left(r+e^{-p} * 0\right)+\frac{\left[r+e^{-p} * 0, r+e^{-p} * 0\right]}{2} \\
& =d r+d\left(e^{-p} * 0\right)+\frac{\left[e^{-p} * 0, e^{-p} * 0\right]}{2}=d r,
\end{aligned}
$$

where the last equality follows from the fact that $e^{-p} * 0$ satisfies the Maurer-Cartan equation in $M \otimes \mathfrak{m}_{A}$. The cohomology class of $(h, r)$ in $H^{2}\left(C_{\chi}\right)$ is well defined and is exactly the obstruction of lifting $\left(x, e^{q}\right)$ to a $\operatorname{MC}_{\chi}(A)$. A straightforward computation shows that the primary obstruction is the quadratic map

$$
H^{1}\left(C_{\chi}\right) \rightarrow H^{2}\left(C_{\chi}\right), \quad(x, a) \mapsto \frac{1}{2}([x, x],[a, \chi(x)]) .
$$

Lemma 2.2. Let $\chi: L \rightarrow M$ be a morphism of DGLA and assume that either

1. $L^{1}=0$, or

2. $\left[L^{1}, L^{1}\right]=0$ and $\left[M^{0}, d M^{0}\right]=0$

Then $\operatorname{Def}_{\chi}$ is smooth.

Proof. In the first case the Maurer-Cartan equation $d l+[l, l] / 2=\chi(l)-e^{-a} * 0=0$ reduces to $e^{-a} * 0=0$ which is equivalent to $d a=0$.

In the second case the Maurer-Cartan equation $d l+[l, l] / 2=\chi(l)-e^{-a} * 0=0$ reduces to $d l=\chi(l)-d a=0$ and then $\mathrm{MC}_{\chi}$ is smooth.

Proposition 2.3. Let

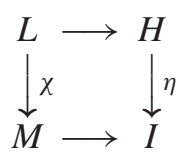

be a commutative diagram of morphisms of differential graded Lie algebras.

If the functor $\operatorname{Def}_{\eta}$ is smooth, then the obstruction space of $\operatorname{Def}_{\chi}$ is contained in the kernel of $H^{2}\left(C_{\chi}\right) \rightarrow H^{2}\left(C_{\eta}\right)$.

Proof. The horizontal arrows are morphisms of DGLA; the explicit description of obstructions given above implies that the two morphism $\operatorname{Def}_{\chi} \rightarrow \operatorname{Def}_{\eta}$ and $H^{2}\left(C_{\chi}\right) \rightarrow H^{2}\left(C_{\eta}\right)$ commute with obstruction maps. The smoothness of Def $\operatorname{Din}_{\eta}$ means that every obstruction vanishes in $H^{2}\left(C_{\eta}\right)$.

Remark 2.4. In the paper [11], written after the first version of this manuscript, we describe an explicit and canonical $L_{\infty}$ structure on $C_{\chi}$ for which the associated Maurer-Cartan and deformation functors coincide with $\mathrm{MC}_{\chi}$ and Def $\mathrm{D}_{\chi}$ respectively. Using this (non trivial) fact it is possible to prove the results of this section using the general theory of $L_{\infty}$-algebras. 


\section{Example: deformations of cohomology groups}

For every pair $V, W$ of graded vector spaces we denote by

$$
\operatorname{Hom}^{*}(V, W)=\bigoplus_{i \in \mathbb{Z}} \operatorname{Hom}^{i}(V, W)
$$

where

$$
\operatorname{Hom}^{i}(V, W)=\left\{f: V \rightarrow W \mid f\left(V_{j}\right) \subset W_{i+j}\right\}=\prod_{j} \operatorname{Hom}\left(V_{j}, W_{i+j}\right) .
$$

If $\left(V, d_{V}\right)$ and $\left(W, d_{W}\right)$ are differential graded vector spaces, then $\operatorname{Hom}^{*}(V, W)$ has a natural differential

$$
\delta: \operatorname{Hom}^{i}(V, W) \rightarrow \operatorname{Hom}^{i+1}(V, W), \quad \delta(f)=d_{W} f-(-1)^{\operatorname{deg}(f)} f d_{V} .
$$

Every $f \in \operatorname{Hom}^{i}(V, W)$ such that $\delta(f)=0$ induces naturally a morphism $H(f) \in$ $\operatorname{Hom}^{i}\left(H^{*}(V), H^{*}(W)\right)$ and for every $i$ the natural transformation

$$
H: H^{i}\left(\operatorname{Hom}^{*}(V, W)\right) \longrightarrow \operatorname{Hom}^{i}\left(H^{*}(V), H^{*}(W)\right)
$$

is an isomorphism. Every pair of morphisms of complexes $p: V^{\prime} \rightarrow V, q: W \rightarrow$ $W^{\prime}$ induces a morphism of differential graded vector spaces

$$
r: \operatorname{Hom}^{*}(V, W) \rightarrow \operatorname{Hom}^{*}\left(V^{\prime}, W^{\prime}\right) .
$$

Notice that if $p, q$ are quasiisomorphisms, then $r$ is a quasiisomorphism. Given a differential graded vector space $(V, d)$, the spaces

$$
\operatorname{Hom}^{*}(V, V), \quad \operatorname{Hom}^{+}(V)=\bigoplus_{i \geq 0} \operatorname{Hom}^{i}(V, V)
$$

are differential graded Lie algebras with bracket

$$
[f, g]:=f \circ g-(-1)^{\operatorname{deg}(f) \operatorname{deg}(g)} g \circ f .
$$

Note that $\delta(f)=[d, f]$ and $f \in \operatorname{Hom}^{1}(V, V)$ satisfies the Maurer-Cartan equation if and only if $(d+f)^{2}=0$.

Lemma 3.1. If $V, W$ are quasiisomorphic complexes of $\mathbb{C}$-vector spaces, then $\operatorname{Hom}^{*}(V, V)$ and $\operatorname{Hom}^{*}(W, W)$ are quasiisomorphic as differential graded Lie algebras.

Proof. Since every complex of vector spaces contains its cohomology as a subcomplex, it is not restrictive to assume $V \subset W$ a subcomplex. 
The subspace $K=\left\{f \in \operatorname{Hom}^{*}(W, W) \mid f(V) \subset V\right\}$ is a differential graded Lie subalgebra and there exists a natural morphism of DGLA $K \rightarrow \operatorname{Hom}^{*}(V, V)$. There exists a commutative diagram of complexes with exact rows

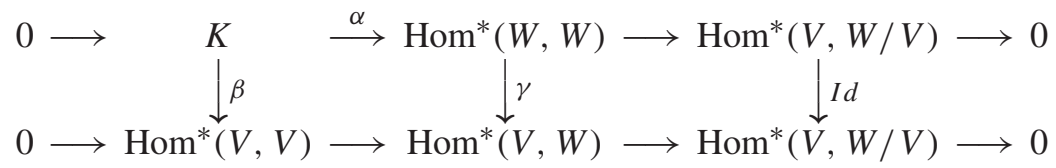

Since $\operatorname{Hom}^{*}(V, W / V)$ is acyclic and $\gamma$ is a quasiisomorphism, it follows that also $\alpha$ and $\beta$ are quasi-isomorphisms.

It is clear that the inclusion of DGLA's $\operatorname{Hom}^{+}(V, V) \subset \operatorname{Hom}^{*}(V, V)$ induces an isomorphism of deformation functors. Denoting, for notational simplicity, $L=$ $\operatorname{Hom}^{+}(V, V)$, for every local Artinian $\mathbb{C}$-algebra $(A, \mathfrak{m})$, the Maurer-Cartan elements $\operatorname{MC}_{L}(A)$ are exactly the deformations of the differential $d$ over $A$, while the group $\exp \left(L^{0} \otimes \mathfrak{m}\right)$ is the group of automorphisms of the graded $A$-module $V \otimes A$ lifting the identity on $V$.

Definition 3.2. We shall say that $x \in \mathrm{MC}_{L}(A)$ gives a deformation of $H^{i}(V, d)$ if the cohomology group $H^{i}(V \otimes A, d+x)$ is a flat $A$-module and the projection onto the residue field induces an isomorphism $H^{i}(V \otimes A, d+x) \otimes_{A} \mathbb{C} \simeq H^{i}(V, d)$.

It is clear that the subset of $x \in \operatorname{MC}_{L}(A)$ giving a deformation of $H^{i}(V, d)$ is stable under the gauge action. We recall that every flat module over an Artinian ring is free.

Lemma 3.3. In the notation above, if $H^{i-1}(V \otimes A, d+x)$ and $H^{i+1}(V \otimes A, d+x)$ are deformations of $H^{i-1}(V, d)$ and $H^{i+1}(V, d)$ respectively, then also $H^{i}(V \otimes$ $A, d+x)$ is a deformation of $H^{i}(V, d)$.

Proof. This is standard (see e.g. [1]).

Lemma 3.4. In the notation above, assume that the complex $(V, d)$ is bounded, i.e.

$$
(V, d): \quad 0 \longrightarrow V^{m} \stackrel{d}{\longrightarrow} V^{m+1} \stackrel{d}{\longrightarrow} \cdots \stackrel{d}{\longrightarrow} V^{n} \longrightarrow 0
$$

for some pair of integers $m \leq n$. Then $x \in M C_{L}(A)$ is gauge equivalent to 0 if and only if the (entire) cohomology of $(V \otimes A, d+x)$ is a deformation of the cohomology of $(V, d)$.

Proof. First we note that every deformation of a vector space over $A$ is trivial and then the cohomology of $(V \otimes A, d+x)$ is a deformation of the cohomology of $(V, d)$ if and only if $H(V \otimes A, d+x) \simeq H(V \otimes A, d)=H(V, d) \otimes A$. By definition, if $a \in L^{0} \otimes \mathfrak{m}$, then $e^{a} * x=e^{a}(d+x) e^{-a}-d$ and therefore $x, y \in M C_{L}(A)$ are gauge equivalent if and only if the complex $(V \otimes A, d+x)$ is isomorphic to $(V \otimes A, d+y)$. In particular if $x$ is gauge equivalent of 0 then the cohomology of $(V \otimes A, d+x)$ is isomorphic to the cohomology of $(V \otimes A, d)$. 
Conversely assume that $x \in M C_{L}(A)$ and $H(V \otimes A, d+x) \simeq H(V \otimes A, d)$; since the complex is bounded, the local flatness criterion implies that there exists an isomorphism of complexes $(V \otimes A, d+x) \simeq(V \otimes A, d)$.

Choosing an index $i$, we consider the truncated complex

$$
\left(T_{i} V, d\right): \quad V^{i-1} \stackrel{d}{\longrightarrow} V^{i} \stackrel{d}{\longrightarrow} V^{i+1} .
$$

Then there exists a natural morphism of graded vector spaces

$$
T_{i}: \operatorname{Hom}^{+}(V, V) \rightarrow \operatorname{Hom}^{*}\left(T_{i} V, T_{i} V\right)
$$

induced by the inclusion $T_{i} V \subset V$ and the projection $V \rightarrow T_{i} V$. It is straightforward to check that $T_{i}$ is a morphism of DGLA; moreover the commutative diagram

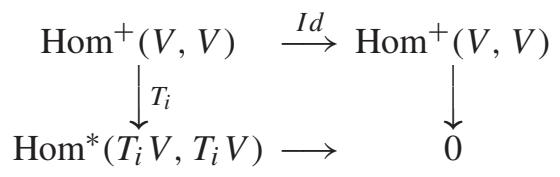

induces a natural transformation of functors $\operatorname{Def}_{T_{i}} \rightarrow \operatorname{Def}_{L}$.

Proposition 3.5. In the notation above, assume there is given a deformation of the complex $x \in M C_{L}(A)$. The class $[x] \in \operatorname{Def}_{L}(A)$ belongs to the image of $\operatorname{Def}_{T_{i}}(A) \rightarrow \operatorname{Def}_{L}(A)$ if and only if the cohomology group $H^{i}(V \otimes A, d+x)$ is a deformation of $H^{i}(V, d)$.

Proof. By definition, the image of $\operatorname{Def}_{T_{i}}(A) \rightarrow \operatorname{Def}_{L}(A)$ is the kernel of

$$
T_{i}: \operatorname{Def}_{L}(A) \rightarrow \operatorname{Def}_{\mathrm{Hom}}{\left(T_{i} V, T_{i} V\right)}(A)
$$

The proof is an immediate consequence of Lemma 3.4 and Lemma 3.3.

\section{Example: Brill-Noether functors of vector bundles}

Let $E, F$ be holomorphic vector bundles on a compact complex manifold $X$ of dimension $n$. We want to describe the deformations $E_{t}$ of $E$ such that $\operatorname{dim} H^{i}(F \otimes$ $\left.E_{t}\right)=\operatorname{dim} H^{i}(F \otimes E)$ for every index $i$. We have seen that the infinitesimal deformations of $E$ are governed by the differential graded Lie algebra

$$
L=\oplus_{i \geq 0} L^{i}, \quad L^{i}=A_{X}^{0, i}(\operatorname{End}(E)) .
$$

This means that for every $(B, \mathfrak{n}) \in \mathbf{A r t}$

$$
\operatorname{Def}_{L}(B) \simeq\left\{E_{B} \rightarrow X \times \operatorname{Spec}(B) \mid E_{B} \text { is a deformation of } E\right\} / \sim
$$


where $\sim$ denotes isomorphism of deformations. Our attention is devoted to the subfunctor $\mathcal{F} \subset \operatorname{Def}_{L}$,

$\mathcal{F}(B)=\left\{E_{B} \rightarrow X \times \operatorname{Spec}(B) \mid H^{i}\left(F \otimes E_{B}\right)\right.$ is a deformation of $\left.H^{i}(F \otimes E)\right\} / \sim$.

Every $x \in L^{i}$ induces naturally morphisms of sheaves

$$
x: \mathcal{A}_{X}^{0, j}(F \otimes E) \rightarrow \mathcal{A}_{X}^{0, i+j}(F \otimes E) .
$$

Denoting by

$\left(A_{X}(F \otimes E), \bar{\partial}\right): 0 \longrightarrow A_{X}^{0,0}(F \otimes E) \stackrel{\bar{\partial}}{\longrightarrow} A_{X}^{0,1}(F \otimes E) \stackrel{\bar{\partial}}{\longrightarrow} \cdots \stackrel{\bar{\partial}}{\longrightarrow} A_{X}^{0, n}(F \otimes E) \longrightarrow 0$

the Dolbeault complex of $F \otimes E$, there exists a natural morphism of DGLA

$$
\chi: L \longrightarrow \operatorname{Hom}^{*}(A(F \otimes E), A(F \otimes E)) .
$$

For every $x \in \operatorname{MC}_{L}(B)$ we denote by $E_{B, x} \rightarrow X \times \operatorname{Spec}(B)$ the corresponding deformation of $E$. We recall that

$$
\mathcal{O}\left(F \otimes E_{B, x}\right)=\operatorname{ker}\left(\mathcal{A}_{X}^{0,0}(F \otimes E) \otimes B \stackrel{\bar{\partial}+x}{\longrightarrow} \mathcal{A}_{X}^{0,1}(F \otimes E) \otimes B\right)
$$

and then the complex of sheaves

$$
\mathcal{A}_{X}^{0,0}(F \otimes E) \otimes B \stackrel{\bar{\partial}+x}{\longrightarrow} \mathcal{A}_{X}^{0,1}(F \otimes E) \otimes B \stackrel{\bar{\partial}+x}{\longrightarrow} \mathcal{A}_{X}^{0,2}(F \otimes E) \otimes B \stackrel{\bar{\partial}+x}{\longrightarrow} \cdots
$$

is a fine resolution of $F \otimes E_{B, x}$. As a consequence, the cohomology of $F \otimes E_{B, x}$ is isomorphic to the cohomology of the complex of free $B$-modules

$$
A_{X}^{0,0}(F \otimes E) \otimes B \stackrel{\bar{\partial}+x}{\longrightarrow} A_{X}^{0,1}(F \otimes E) \otimes B \stackrel{\bar{\partial}+x}{\longrightarrow} A_{X}^{0,2}(F \otimes E) \otimes B \stackrel{\bar{\partial}+x}{\longrightarrow} \cdots
$$

Lemma 4.1. Consider the morphism of differential graded Lie algebras

$$
L:=A_{X}^{0, *}(\operatorname{End}(E)) \stackrel{\chi}{\longrightarrow} \operatorname{Hom}^{*}\left(A_{X}(F \otimes E), A_{X}(F \otimes E)\right) .
$$

Then the subfunctor $\mathcal{F}$ is the image of $\operatorname{Def}_{\chi} \rightarrow \operatorname{Def}_{L}$. In particular, the tangent space of $\mathcal{F}$ is the kernel of the natural map

$$
H^{1}(\operatorname{End}(E)) \rightarrow \bigoplus_{i} \operatorname{Hom}\left(H^{i-1}(F \otimes E), H^{i}(F \otimes E)\right) \text {. }
$$

Proof. The first part is an immediate consequence of the results of the previous sections. The tangent space is the kernel of

$$
H^{1}(\operatorname{End}(E)) \rightarrow H^{1}\left(\operatorname{Hom}^{*}\left(A_{X}(F \otimes E), A_{X}(F \otimes E)\right)\right) .
$$

On the other hand, according to Lemma 3.1 we have

$$
H^{1}\left(\operatorname{Hom}^{*}\left(A_{X}(F \otimes E), A_{X}(F \otimes E)\right)\right)=\oplus_{i} \operatorname{Hom}\left(H^{i-1}(F \otimes E), H^{i}(F \otimes E)\right) .
$$


As an application we get a new proof of the following smoothness theorem of Green and Lazarsfeld [16].

Theorem 4.2. In the notation above, if $X$ is compact Kähler, $E \in \mathrm{Pic}^{0}(X)$ and $F$ is a flat unitary bundle, then $\operatorname{Def}_{\chi}$ is smooth.

Proof. We first point out that, if $G$ is a flat unitary vector bundle on $X$, then it makes sense to consider the sheaves $\bar{\Omega}_{X}^{i}(G)=\operatorname{ker}(\partial)$ of $G$-valued antiholomorphic differential forms and, by Hodge theory on $G$, the inclusion of complexes

$$
\left(\Gamma\left(X, \bar{\Omega}_{X}^{*}(G)\right), 0\right) \subset\left(A_{X}^{0, *}(G), \bar{\partial}\right),
$$

is an injective quasiisomorphism. Since $E$ is flat unitary it is not restrictive to assume $E=\mathcal{O}_{X}$; the differential graded Lie algebra $L$ is then Abelian and isomorphic to the complex $\left(A_{X}^{0, *}, \bar{\partial}\right)$. Denoting by

$$
M=\left\{a \in \operatorname{Hom}^{*}\left(A_{X}(F), A_{X}(F)\right) \mid a\left(\Gamma\left(X, \bar{\Omega}_{X}^{*}(F)\right)\right) \subset \Gamma\left(X, \bar{\Omega}_{X}^{*}(F)\right)\right\}
$$

we have a commutative diagram of morphisms of DGLA

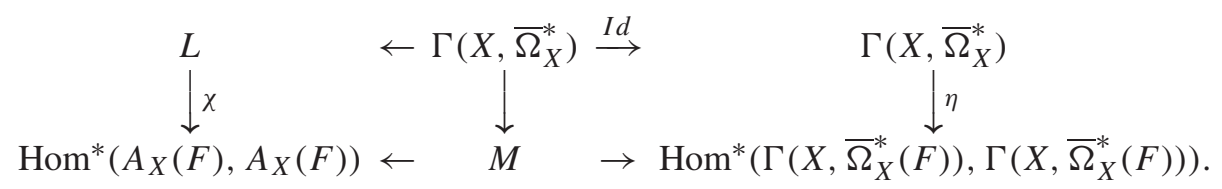

The horizontal arrows are quasiisomorphisms and therefore $\operatorname{Def}_{\chi}=\operatorname{Def}_{\eta}$ by Theorem 2.1. Then, according to Lemma 2.2, the functor $\operatorname{Def}_{\eta}$ is smooth.

\section{Example: the Hilbert functor of a smooth submanifold}

Let $X$ be a smooth complex manifold of dimension $n$ and denote by

$$
\operatorname{Der}^{p}\left(\mathcal{A}_{X}^{0, *}, \mathcal{A}_{X}^{0, *}\right)
$$

the sheaf of $\mathbb{C}$-derivations of degree $p$ of the sheaf of graded algebras $\left(\mathcal{A}_{X}^{0, *}, \wedge\right)$ (note that $\bar{\partial} \in \operatorname{Der}^{1}\left(\mathcal{A}_{X}^{0, *}, \mathcal{A}_{X}^{0, *}\right)$ ).

The DGLA structure on the sheaf $\operatorname{Der}^{*}\left(\mathcal{A}_{X}^{0, *}, \mathcal{A}_{X}^{0, *}\right)=\oplus_{p} \operatorname{Der}^{p}\left(\mathcal{A}_{X}^{0, *}, \mathcal{A}_{X}^{0, *}\right)$ is induced by the standard bracket

$$
[f, g]:=f \circ g-(-1)^{\operatorname{deg}(f) \operatorname{deg}(g)} g \circ f,
$$

and the differential

$$
d f:=[\bar{\partial}, f]=\bar{\partial} \circ f-(-1)^{\operatorname{deg}(f)} f \circ \bar{\partial} .
$$


For every $p$ we interpret $\mathcal{A}_{X}^{0, p}\left(T_{X}\right)$ as a subsheaf of $\operatorname{Der}^{p}\left(\mathcal{A}_{X}^{0, *}, \mathcal{A}_{X}^{0, *}\right)$, where the inclusion is described in local holomorphic coordinates $z_{1}, \ldots, z_{n}$ by

$$
\left(\phi \frac{\partial}{\partial z_{i}}\right)\left(f d \bar{z}_{j_{1}} \wedge \cdots \wedge d \bar{z}_{j_{k}}\right)=\frac{\partial f}{\partial z_{i}} \phi \wedge d \bar{z}_{j_{1}} \wedge \cdots \wedge d \bar{z}_{j_{k}} .
$$

We note that $\mathcal{A}_{X}^{0, *}\left(T_{X}\right)=\oplus_{p} \mathcal{A}_{X}^{0, p}\left(T_{X}\right)$ is a sheaf of differential graded Lie subalgebras of $\mathcal{D e r}^{*}\left(\mathcal{A}_{X}^{0, *}, \mathcal{A}_{X}^{0, *}\right)$. A straightforward computation shows that, if $z_{1}, \ldots, z_{n}$ are local holomorphic coordinates, $I, J$ ordered subsets of $\{1, \ldots, n\}, a=f d \bar{z}_{I} \frac{\partial}{\partial z_{i}}$, $b=g d \bar{z}_{J} \frac{\partial}{\partial z_{j}}, f, g \in \mathcal{A}_{X}^{0,0}$ then

$$
d a=\bar{\partial} f \wedge d \bar{z}_{I} \frac{\partial}{\partial z_{i}}, \quad[a, b]=d \bar{z}_{I} \wedge d \bar{z}_{J}\left(f \frac{\partial g}{\partial z_{i}} \frac{\partial}{\partial z_{j}}-g \frac{\partial f}{\partial z_{j}} \frac{\partial}{\partial z_{i}}\right) .
$$

Assume now that $i: Z \hookrightarrow X$ is the inclusion of a closed submanifold and denote by

$$
i^{*}:\left(\mathcal{A}_{X}^{0, *}, \bar{\partial}\right) \rightarrow\left(\mathcal{A}_{Z}^{0, *}, \bar{\partial}\right)
$$

the morphism of sheaves of differential graded algebras given by restriction of forms on $Z$. We denote by

$$
\mathcal{A}_{X}^{0, *}\left(T_{X}\right)(-\log Z)=\left\{\eta \in \mathcal{A}_{X}^{0, *}\left(T_{X}\right) \mid \eta\left(\operatorname{ker}\left(i^{*}\right)\right) \subset \operatorname{ker}\left(i^{*}\right)\right\} .
$$

We note that $\mathcal{A}_{X}^{0, *}\left(T_{X}\right)(-\log Z)$ is a sheaf of differential graded Lie subalgebras of $\mathcal{A}^{0, *}\left(T_{X}\right)$ and there exists an exact sequence of fine sheaves

$$
0 \rightarrow \mathcal{A}_{X}^{0, *}\left(T_{X}\right)(-\log Z) \rightarrow \mathcal{A}_{X}^{0, *}\left(T_{X}\right) \rightarrow \mathcal{A}_{Z}^{0, *}\left(N_{Z \mid X}\right) \rightarrow 0 .
$$

Let $(A, \mathfrak{m})$ be a local Artinian $\mathbb{C}$-algebra, every $\eta \in \mathcal{A}_{X}^{0,0}\left(T_{X}\right) \otimes \mathfrak{m}$ induces an automorphism

$$
e^{\eta}: \mathcal{A}_{X}^{0, *} \otimes A \rightarrow \mathcal{A}_{X}^{0, *} \otimes A, \quad e^{\eta}(h)=\sum_{n=0}^{\infty} \frac{\eta^{n}}{n !}(h) .
$$

If $\eta \in \mathcal{A}_{X}^{0,0}\left(T_{X}\right)(-\log Z) \otimes \mathfrak{m}$, then $e^{\eta}\left(\operatorname{ker}\left(i^{*}\right) \otimes A\right)=\operatorname{ker}\left(i^{*}\right) \otimes A$.

Lemma 5.1. For every local Artinian $\mathbb{C}$-algebra $(A, \mathfrak{m})$ and every $\eta \in \mathcal{A}^{0,0}\left(T_{X}\right) \otimes$ $\mathfrak{m}$ we have

$$
e^{\eta} \circ \bar{\partial} \circ e^{-\eta}=\bar{\partial}+e^{\eta} * 0: \mathcal{A}_{X}^{0,0} \otimes A \rightarrow \mathcal{A}_{X}^{0,1} \otimes A,
$$

where $*$ is the gauge action on $\mathcal{A}_{X}^{0, *}\left(T_{X}\right) \otimes \mathfrak{m}$. In particular

$$
\operatorname{ker}\left(\bar{\partial}+e^{\eta} * 0: \mathcal{A}_{X}^{0,0} \otimes A \rightarrow \mathcal{A}_{X}^{0,1} \otimes A\right)=e^{\eta}\left(\mathcal{O}_{X} \otimes A\right) .
$$


Proof. This follows from the definition of the gauge action and the fact that $\mathcal{A}_{X}^{0, *}\left(T_{X}\right)$ is a subalgebra of $\operatorname{Der}^{*}\left(\mathcal{A}_{X}^{0, *}, \mathcal{A}_{X}^{0, *}\right)$.

We denote by $A_{X}^{0, *}\left(T_{X}\right)(-\log Z)$ the differential graded Lie algebras of global sections of the sheaf $\mathcal{A}_{X}^{0, *}\left(T_{X}\right)(-\log Z)$, while, according to our general notation, we denote by $A_{X}^{0, *}\left(T_{X}\right)$ the DGLA of global sections of $\mathcal{A}_{X}^{0, *}\left(T_{X}\right)$. The differential graded Lie algebra $A_{X}^{0, *}\left(T_{X}\right)$ is called the Kodaira-Spencer algebra of $X$.

The natural inclusion $\chi: A_{X}^{0, *}\left(T_{X}\right)(-\log Z) \rightarrow A_{X}^{0, *}\left(T_{X}\right)$ is a morphism of differential graded Lie algebras and its cokernel is isomorphic to the Dolbeault complex of $N_{Z \mid X}$; in particular for every $i \geq 0$

$$
H^{i}\left(Z, N_{Z \mid X}\right) \simeq H^{i}\left(A_{X}^{0, *}\left(T_{X}\right) / A_{X}^{0, *}\left(T_{X}\right)(-\log Z)\right) \simeq H^{i+1}\left(C_{\chi}\right) .
$$

In the sequel of this section, just to avoid heavy formulas, we denote by $L_{Z \mid X}$ the differential graded Lie algebra $A_{X}^{0, *}\left(T_{X}\right)(-\log Z)$.

Consider now the associated functor $\operatorname{Def}_{\chi}$. Since $\chi$ is injective we have, for every local Artinian $\mathbb{C}$-algebra $(A, \mathfrak{m})$,

$$
\operatorname{MC}_{\chi}(A)=\left\{e^{\eta} \in \operatorname{Aut}_{A}\left(\mathcal{A}_{X}^{0,0} \otimes A\right) \mid \eta \in A_{X}^{0,0}\left(T_{X}\right) \otimes \mathfrak{m}, e^{-\eta} * 0 \in L_{Z \mid X}^{1} \otimes \mathfrak{m}\right\} .
$$

Under this identification the gauge action becomes

$$
\exp \left(L_{Z \mid X}^{0} \otimes \mathfrak{m}\right) \times \operatorname{MC}_{\chi}(A) \rightarrow \operatorname{MC}_{\chi}(A), \quad\left(e^{\mu}, e^{\eta}\right) \mapsto e^{\eta} \circ e^{-\mu},
$$

and then

$$
\operatorname{Def}_{\chi}(A)=\frac{\operatorname{MC}_{\chi}(A)}{\exp \left(L_{Z \mid X}^{0} \otimes \mathfrak{m}\right)} .
$$

Theorem 5.2. There exists an isomorphism of functors $\theta: \operatorname{Def}_{\chi} \rightarrow \operatorname{Hilb}_{X}^{Z}$.

Proof. This is implicitly proved in [5, Section 2] and [6] using the theory of transversely holomorphic trivialization (in the relative case). Here we sketch a different proof.

Denote by $\mathcal{I}=\mathcal{O}_{X} \cap \operatorname{ker}\left(i^{*}\right)$ the holomorphic ideal sheaf of $Z$ and define

$\theta: \operatorname{Def}_{\chi}(A) \rightarrow$ ideal sheaves of $\left.\mathcal{O}_{X} \otimes \mathbb{C} A\right\}, \quad \theta\left(e^{\eta}\right)=\left(\mathcal{O}_{X} \otimes A\right) \cap e^{\eta}\left(\operatorname{ker}\left(i^{*}\right) \otimes A\right)$.

The morphism $\theta$ is well defined because, if $\mu \in L_{Z \mid X}^{0} \otimes \mathfrak{m}$, then

$\theta\left(e^{\eta} \circ e^{-\mu}\right)=\left(\mathcal{O}_{X} \otimes A\right) \cap e^{\eta}\left(e^{-\mu}\left(\operatorname{ker}\left(i^{*}\right) \otimes A\right)\right)=\left(\mathcal{O}_{X} \otimes A\right) \cap e^{\eta}\left(\operatorname{ker}\left(i^{*}\right) \otimes A\right)$.

Next, we need to prove that $\theta\left(e^{\eta}\right)$ is flat over $A$ and $\theta\left(e^{\eta}\right) \otimes_{A} \mathbb{C}=\mathcal{I}$; clearly we can prove the same properties for the sheaf $e^{-\eta}\left(\theta\left(e^{\eta}\right)\right)$. According to Lemma 5.1

$$
e^{-\eta}\left(\mathcal{O}_{X} \otimes A\right)=\operatorname{ker}\left(\bar{\partial}+e^{-\eta} * 0: \mathcal{A}_{X}^{0,0} \otimes A \rightarrow \mathcal{A}_{X}^{0,1} \otimes A\right),
$$


and then

$$
e^{-\eta}\left(\theta\left(e^{\eta}\right)\right)=\left(\operatorname{ker}\left(i^{*}\right) \otimes A\right) \cap \operatorname{ker}\left(\bar{\partial}+e^{-\eta} * 0\right) .
$$

Since flatness is a local property it is not restrictive to assume $X$ a Stein manifold, $H^{1}\left(X, T_{X}\right)=0$ and $H^{0}\left(X, T_{X}\right) \rightarrow H^{0}\left(Z, N_{Z \mid X}\right)$ surjective. This implies that $H^{1}\left(L_{Z \mid X}\right)=0$ and then the functor $\operatorname{Def}_{L_{Z \mid X}}$ is trivial. In particular there exists $\mu \in L_{Z \mid X}^{0} \otimes \mathfrak{m}$ such that $e^{-\mu} * 0=e^{-\eta} * 0$ and therefore

$$
e^{-\eta}\left(\theta\left(e^{\eta}\right)\right)=\left(\operatorname{ker}\left(i^{*}\right) \otimes A\right) \cap \operatorname{ker}\left(\bar{\partial}+e^{-\eta} * 0\right)=e^{-\mu}\left(\theta\left(e^{\mu}\right)\right)=e^{-\mu}(\mathcal{I} \otimes A) .
$$

This proves that $\theta: \operatorname{Def}_{\chi} \rightarrow \operatorname{Hilb}_{X}^{Z}$. It is well known (see e.g. [23]) that the functor $\mathrm{Hilb}_{X}^{Z}$ is prorepresentable, its tangent space is $H^{0}\left(Z, N_{Z \mid X}\right)$ and its obstructions are in $H^{1}\left(Z, N_{Z \mid X}\right)$. Therefore, in order to prove that $\theta$ is an isomorphism it is sufficient to prove that it is bijective on tangent space and injective on obstruction space. This is a straightforward computation and it is left to the reader.

In analogy with rational homotopy theory (see also next Remark 6.8), it is possible to define the Whitehead product

$$
[,]_{W}: H^{i}\left(Z, N_{Z \mid X}\right) \times H^{j}\left(Z, N_{Z \mid X}\right) \rightarrow H^{i+j+1}\left(Z, N_{Z \mid X}\right)
$$

in the following way. For every cohomology class $a \in H^{i}\left(Z, N_{Z \mid X}\right)$ we denote by $\tilde{a} \in A_{X}^{0, i}\left(T_{X}\right)$ a differential form that lifts $a$. This means in particular that $d \tilde{a} \in L_{Z \mid X}^{i+1}$ and $\tilde{a}$ is defined up to elements of $d A_{X}^{0, i-1}\left(T_{X}\right)+L_{Z \mid X}^{i}$.

Given $a \in H^{i}\left(Z, N_{Z \mid X}\right)$ and $b \in H^{j}\left(Z, N_{Z \mid X}\right)$, we define

$$
[a, b]_{W} \in H^{i+j+1}\left(Z, N_{Z \mid X}\right)
$$

as the cohomology class of $\frac{1}{2}\left([\tilde{a}, d \tilde{b}]-(-1)^{i}[d \tilde{a}, \tilde{b}]\right)$. It is easy to verify that $[,]_{W}$ is well defined and induces a structure of graded Lie algebra on the space $\oplus V^{i}, V^{i}=H^{i-1}\left(Z, N_{Z \mid X}\right)$.

Corollary 5.3. The primary obstruction map of $\operatorname{Hilb}_{X}^{Z}$ is equal to

$$
H^{0}\left(Z, N_{Z \mid X}\right) \rightarrow H^{1}\left(Z, N_{Z \mid X}\right), \quad a \mapsto \frac{1}{2}[a, a]_{W}
$$

Proof. This follows from the description of the primary obstruction map of the functor $\operatorname{Def}_{\chi}$.

\section{Extended deformations}

In this section we shall prove Theorem 2.1 by using general properties of extended deformation functors (EDF), introduced in [26].

We first recall the definition and the main properties of extended deformation functors. We denote by: 
- $\mathbf{C}$ the category of all nilpotent finite dimensional dg-algebras over $\mathbb{C}$.

- $\mathbf{C}_{0}$ the full subcategory of $\mathbf{C}$ whose objects are the dg-algebras $A \in \mathbf{C}$ with trivial multiplication, i.e. $A \cdot A=0$.

In other words an object in $\mathbf{C}$ is a finite dimensional complex $A=\oplus A_{i}$ endowed with a structure of dg-algebra such that $A \cdot A \cdots(n$ factors $) \cdots A=0$ for $n>>0$. Note that if $A=A_{0}$ is concentrated in degree 0 , then $A \in \mathbf{C}$ if and only if $A$ is the maximal ideal of a local Artinian $\mathbb{C}$-algebra with residue field $\mathbb{C}$.

There is an obvious equivalence between $\mathbf{C}_{0}$ and the category of finite dimensional complexes of $\mathbb{C}$-vector spaces. If $A \in \mathbf{C}$ and $I \subset A$ is a differential ideal, then also $I \in \mathbf{C}$ and the inclusion $I \rightarrow A$ is a morphism of dg-algebras.

Definition 6.1. A small extension in $\mathbf{C}$ is a short exact sequence of complexes

$$
0 \longrightarrow I \longrightarrow A \stackrel{\alpha}{\longrightarrow} B \longrightarrow 0
$$

such that $\alpha$ is a morphism in $\mathbf{C}$ and $I$ is an ideal of $A$ such that $A I=0$; in addition it is called acyclic if $I$ is an acyclic complex, or equivalently if $\alpha$ is a quasiisomorphism.

Definition 6.2. A covariant functor $F: \mathbf{C} \rightarrow$ Set is called a predeformation functor if the following conditions are satisfied:

6.2.1. $F(0)=\{$ one element $\}$.

6.2.2. For every $A, B \in \mathbf{C}$, the natural map

$$
F(A \times B) \rightarrow F(A) \times F(B)
$$

is bijective.

6.2.3. For every surjective morphism $\alpha: A \rightarrow C$ in $\mathbf{C}$, with $C \in \mathbf{C}_{0}$ an acyclic complex, the natural morphism

$$
F(\operatorname{ker}(\alpha)) \rightarrow F(A)
$$

is bijective.

6.2.4. For every pair of morphisms $\alpha: A \rightarrow C, \beta: B \rightarrow C$ in $\mathbf{C}$, with $\alpha$ surjective, the natural map

$$
F\left(A \times_{C} B\right) \rightarrow F(A) \times{ }_{F(C)} F(B)
$$

is surjective.

6.2.5. For every acyclic small extension

$$
0 \longrightarrow I \longrightarrow A \longrightarrow B \longrightarrow 0
$$

the induced map $F(A) \rightarrow F(B)$ is surjective.

Definition 6.3. A covariant functor $F: \mathbf{C} \rightarrow$ Set is called a deformation functor if it is a predeformation functor and $F(I)=0$ for every acyclic complex $I \in \mathbf{C}_{0}$. 
Example 6.4. Let $L$ be a differential graded Lie algebra and $A \in \mathbf{C}$; then the tensor product $L \otimes A$ has a natural structure of nilpotent DGLA with

$$
\begin{aligned}
(L \otimes A)^{i} & =\oplus_{j \in \mathbb{Z}} L^{j} \otimes A_{i-j} \\
d(x \otimes a) & =d x \otimes a+(-1)^{\operatorname{deg}(x)} x \otimes d a \\
{[x \otimes a, y \otimes b] } & =(-1)^{\operatorname{deg}(a) \operatorname{deg}(y)}[x, y] \otimes a b .
\end{aligned}
$$

Every morphism of DGLA, $L \rightarrow N$ and every morphism $A \rightarrow B$ in $\mathbf{C}$ give a natural commutative diagram of morphisms of differential graded Lie algebras

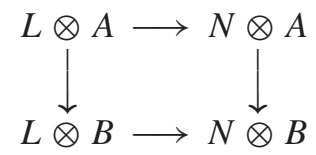

The (extended) exponential functor $\widetilde{\exp }_{L}: \mathbf{C} \rightarrow$ Set is defined as

$$
\widetilde{\exp }_{L}(A)=\exp \left(H^{0}(L \otimes A)\right)
$$

It is a deformation functor in the sense of 6.3.

Example 6.5. The (extended) deformation governed by $L$ is by definition given by the functor

$$
\widetilde{\operatorname{Def}}_{L}: \mathbf{C} \rightarrow \text { Set, } \quad \widetilde{\operatorname{Def}}_{L}(A)=\frac{\left\{x \in(L \otimes A)^{1} \mid d x+[x, x] / 2=0\right\}}{\text { Gauge action of } \exp \left((L \otimes A)^{0}\right)} .
$$

It is proved in [26] that $\widetilde{\operatorname{Def}_{L}}$ is a deformation functor in the sense of Definition 6.3.

For every predeformation functor $F$ and every $A \in \mathbf{C}_{0}$ there exists a natural structure of vector space on $F(A)$, where the sum and the scalar multiplication are described by the maps

$$
\begin{gathered}
A \times A \stackrel{+}{\longrightarrow} A \Rightarrow F(A \times A)=F(A) \times F(A) \stackrel{+}{\longrightarrow} F(A) \\
s \in \mathbb{C}, \quad A \stackrel{\cdot s}{\longrightarrow} A \quad \Rightarrow \quad F(A) \stackrel{\cdot s}{\longrightarrow} F(A) .
\end{gathered}
$$

If $A \rightarrow B$ is a morphism in $\mathbf{C}_{0}$, then $F(A) \rightarrow F(B)$ is $\mathbb{C}$-linear. Similarly if $F \rightarrow G$ is a natural transformations of predeformation functors, the map $F(A) \rightarrow G(A)$ is $\mathbb{C}$-linear for every $A \in \mathbf{C}_{0}$.

Definition 6.6. Let $F$ be a deformation functor and denote $T F[1]^{n}=T^{n+1} F=$ $F(\mathbb{C} \epsilon)$, where $\epsilon$ is an indeterminate of degree $-n \in \mathbb{Z}$ such that $\epsilon^{2}=0$. The graded vector space $T F[1]=\bigoplus_{n \in \mathbb{Z}} T F[1]^{n}$ is called the tangent space of $F$.

A natural transformation $F \rightarrow G$ of deformation functors is called a quasiisomorphism if induces an isomorphism on tangent spaces, i.e. if $T^{n} F \rightarrow T^{n} G$ is bijective for every $n$. 
For example, if $L$ is a differential graded Lie algebra, then $T^{n} \widetilde{\exp }_{L}=H^{n-1}(L)$ and $T^{n} \widetilde{\operatorname{Def}}_{L}=H^{n}(L)$.

Theorem 6.7 (Inverse function theorem). A natural transformation of deformation functors is an isomorphism if and only if it is a quasiisomorphism.

Proof. See [26, Corollary 3.2] or [28, Corollary 5.72].

Remark 6.8. It is proved in [26] that for every deformation functor $F$ there is defined the Whitehead product on its tangent space

$$
[,]_{W}: T F[1]^{i} \times T F[1]^{j} \rightarrow T F[1]^{i+j+1}
$$

inducing a graded Lie algebra structure on $\oplus T^{n} F$. As in the topological case (see e.g. [34, page 111]), the product $[,]_{W}$ measure the obstruction to lifting a map from a wedge (of spheres in topology and of dg-fat points in deformation theory) to a product. In the topological analogy, the space $\pi_{i}(X) \otimes \mathbb{Q}$ corresponds to $T F[1]^{-i}$, where $F=\operatorname{Mor}(-, X)$.

Our next goal is to interpret the functors $\widetilde{\exp }_{L}$ and $\widetilde{\operatorname{Def}}_{L}$ as special cases of a suitable functor $\widetilde{\operatorname{Def}_{\chi}}$, where $\chi$ is a morphism of differential graded Lie algebras.

Definition 6.9. Let $\chi: L \rightarrow M$ be a morphism of differential graded Lie algebras. The (extended) Maurer-Cartan functor $\mathrm{MC}_{\chi}: \mathbf{C} \rightarrow$ Set is defined as

$$
\operatorname{MC}_{\chi}(A)=\left\{\left(x, e^{a}\right) \in(L \otimes A)^{1} \times \exp \left((M \otimes A)^{0}\right) \mid d x+\frac{1}{2}[x, x]=0, e^{a} * \chi(x)=0\right\}
$$

where $*$ is the gauge action of $\exp \left((M \otimes A)^{0}\right)$ on $\mathrm{MC}_{\chi}(A)$

$$
e^{a} * w=w+\sum_{n \geq 0} \frac{[a,-]^{n}}{(n+1) !}([a, w]-d a) .
$$

Lemma 6.10. $\mathrm{MC}_{\chi}$ is a predeformation functor.

Proof. It is evident that $\mathrm{MC}_{\chi}(0)=0$ and for every pair of morphisms $\alpha: A \rightarrow C$, $\beta: B \rightarrow C$ in $\mathbf{C}$ we have

$$
\operatorname{MC}_{\chi}\left(A \times_{C} B\right)=\operatorname{MC}_{\chi}(A) \times \operatorname{MC}_{\chi}(C) \operatorname{MC}_{\chi}(B)
$$

and then $\mathrm{MC}_{\chi}$ satisfies properties 6.2.1, 6.2.2, 6.2.3 and 6.2.4.

Let $0 \longrightarrow I \longrightarrow A \stackrel{\alpha}{\longrightarrow} B \longrightarrow 0$ be an acyclic small extension and $\left(x, e^{q}\right) \in \operatorname{MC}_{\chi}(B)$. Since $\alpha$ is surjective there exists a pair $\left(y, e^{p}\right) \in(L \otimes A)^{1} \times \exp \left((M \otimes A)^{0}\right)$ such that $\alpha(y)=x$ and $\alpha(p)=q$. Setting

$$
h=d y+\frac{1}{2}[y, y] \in(L \otimes I)^{2}
$$


we have

$$
d h=\frac{1}{2} d[y, y]=[d y, y]=[h, y]-\frac{1}{2}[[y, y], y] .
$$

By Jacobi identity $[[y, y], y]=0$, while $[h, y]=0$ because $A I=0$; therefore $d h=0$ and, since the complex $L \otimes I$ is acyclic by Künneth formula, there exists $s \in(L \otimes I)^{1}$ such that $d s=h$. The element $\hat{x}=y-s$ lifts $x$ and satisfies the Maurer-Cartan equation. The element $z=e^{p} * \chi(\hat{x})$ belongs to $M \otimes I$ and satisfies Maurer-Cartan equation. This means that $d z=0$ and then, since $M \otimes I$ is acyclic, there exists $r \in(M \otimes I)^{0}$ such that

$$
e^{r} e^{p} * \chi(\hat{x})=e^{r} * z=z-d r=0
$$

and then $\left(\hat{x}, e^{r} e^{p}\right) \in \operatorname{MC}_{\chi}(A)$ is a lifting of $\left(x, e^{q}\right)$.

Definition 6.11. The functor $\widetilde{\operatorname{Def}_{\chi}}: \mathbf{C} \rightarrow$ Set is the quotient $\widetilde{\operatorname{Def}}_{\chi}(A)=\operatorname{MC}_{\chi}(A) / \sim$, where

$$
\left(x, e^{q}\right) \sim\left(e^{a} * x, e^{d b} e^{q} e^{-\chi(a)}\right), \quad a \in(L \otimes A)^{0}, \quad b \in(M \otimes A)^{-1} .
$$

In other words, $\widetilde{\operatorname{Def}}_{\chi}(A)$ is the set of orbits of the action

$$
\begin{gathered}
\left(\exp \left((L \otimes A)^{0}\right) \times \exp \left(d(M \otimes A)^{-1}\right)\right) \times M C_{\chi}(A) \rightarrow M C_{\chi}(A) \\
\left(\left(e^{a}, e^{d b}\right),\left(x, e^{q}\right)\right) \mapsto\left(e^{a} * x, e^{d b} e^{q} e^{-\chi(a)}\right) .
\end{gathered}
$$

Notice that $\left.\exp \left(d(M \otimes A)^{-1}\right)\right)$ is the irrelevant stabilizer of 0 . We have:

- If $M=0$, then $\widetilde{\operatorname{Def}_{\chi}}=\widetilde{\operatorname{Def}}_{L}$.

- If $L=0$, then $\widetilde{\operatorname{Def}}_{\chi}=\widetilde{\exp }_{M}$.

Theorem 6.12. $\widetilde{\operatorname{Def}}_{\chi}: \mathbf{C} \rightarrow$ Set $i$ s a deformation functor with $T^{i} \widetilde{\operatorname{Def}}_{\chi}=H^{i}\left(C_{\chi}\right)$.

Proof. If $C \in \mathbf{C}_{0}$ then $L \otimes C$ and $M \otimes C$ are Abelian differential graded Lie algebras and

$$
\begin{gathered}
\operatorname{MC}_{\chi}(C)=\left\{\left(x, e^{m}\right) \mid d x=0, \chi(x)-d m=0\right\}, \\
\widetilde{\operatorname{Def}_{\chi}}(C)=\frac{\left\{\left(x, e^{m}\right) \mid d x=0, \chi(x)-d m=0\right\}}{\left\{\left(-d y, e^{d b-\chi(y)}\right) \mid y \in(L \otimes C)^{0}, b \in(M \otimes C)^{-1}\right\}} .
\end{gathered}
$$

Therefore $\widetilde{\operatorname{Def}}_{\chi}(C)$ is isomorphic to the $H^{1}$ of the suspended mapping cone of $\chi: L \otimes C \rightarrow M \otimes C$. If $C$ is acyclic then $\widetilde{\operatorname{Def}}_{\chi}(C)=0$; we have proved that $\widetilde{\operatorname{Def}_{\chi}}$ satisfies the second condition of Definition 6.3 and remains to prove that $\widetilde{\operatorname{Def}_{\chi}}$ is a predeformation functor. Since $\widetilde{\operatorname{Def}}_{\chi}$ is the quotient of the predeformation functor $\mathrm{MC}_{\chi}$, the conditions 6.2.1 and 6.2.5 are trivially verified. It is clear from the definition that $\widetilde{\operatorname{Def}_{\chi}}(A \times B)=\widetilde{\operatorname{Def}}_{\chi}(A) \times \widetilde{\operatorname{Def}}_{\chi}(B)$

Verification of 6.2.4. Let $\alpha: A \rightarrow C, \beta: B \rightarrow C$ morphism in $\mathbf{C}$ with $\alpha$ surjective. Assume there are given $\left(a, e^{p}\right) \in \mathrm{MC}_{\chi}(A),\left(b, e^{q}\right) \in \mathrm{MC}_{\chi}(B)$ such that $\alpha\left(a, e^{p}\right)$ 
and $\beta\left(b, e^{q}\right)$ give the same element in $\widetilde{\operatorname{Def}_{\chi}}(C)$; then there exist $u \in(L \otimes C)^{0}$ and $k \in(M \otimes C)^{-1}$ such that

$$
\beta(b)=e^{u} * \alpha(a), \quad \beta\left(e^{q}\right)=e^{d k} \alpha\left(e^{p}\right) e^{-\chi(u)} .
$$

Let $v \in(L \otimes A)^{0}$ be a lifting of $u$ and $h \in(M \otimes A)^{-1}$ be a lifting of $k$.

Replacing $\left(a, e^{p}\right)$ with its gauge equivalent element $\left(e^{v} * a, e^{d h} e^{p} e^{-\chi(v)}\right)$, we may suppose $\alpha\left(a, e^{p}\right)=\beta\left(b, e^{q}\right)$ and then the pair $\left(\left(a, e^{p}\right),\left(b, e^{q}\right)\right)$ lifts to $\operatorname{MC}_{\chi}\left(A \times_{C}\right.$ $B)$ : this proves that the map

$$
\widetilde{\operatorname{Def}}_{\chi}(A \times C B) \rightarrow \widetilde{\operatorname{Def}_{\chi}}(A) \times \widetilde{\operatorname{Def}}_{\chi}(C) \widetilde{\operatorname{Def}_{\chi}}(B)
$$

is surjective.

Verification of 6.2.3. Assume $C \in \mathbf{C}_{0}$ acyclic, $\alpha: A \rightarrow C$ surjective and denote $D=\operatorname{ker}(\alpha)$. Let $\left(a_{1}, e^{p_{1}}\right),\left(a_{2}, e^{p_{2}}\right) \in \mathrm{MC}_{\chi}(D), u \in(L \otimes A)^{0}$ and $k \in(M \otimes A)^{-1}$ be such that $a_{2}=e^{u} * a_{1}$ and $e^{p_{2}}=e^{d k} e^{p_{1}} e^{-\chi(u)}$. We want to prove that there exist $v \in(L \otimes D)^{0}$ and $f \in(M \otimes D)^{-1}$ such that $e^{v} * a_{1}=a_{2}$ and $e^{p_{2}}=e^{d f} e^{p_{1}} e^{-\chi(v)}$.

Since $\alpha\left(a_{1}\right)=\alpha\left(a_{2}\right)=0$ and $L \otimes C$ is an Abelian DGLA we have $0=$ $e^{\alpha(u)} * 0=0-d \alpha(u)$ and then $d \alpha(u)=0$. Since $L \otimes C$ is acyclic there exists $h \in(L \otimes A)^{-1}$ such that $d \alpha(h)=-\alpha(u)$ and $u+d h \in(L \otimes D)^{0}$. Setting $w=\left[a_{1}, h\right]+d h$, then $e^{w}$ belongs to the irrelevant stabilizer of $a_{1}$ and therefore $\left(e^{u} e^{w}\right) * a_{1}=e^{u} * a_{1}=a_{2}$. Writing $e^{u} e^{w}=e^{v}$, we claim that $v \in L \otimes D$ : in fact

$$
v=u \bullet w \equiv u+w \equiv u+d h \quad(\bmod [L \otimes A, L \otimes A])
$$

and since $A \cdot A \subset D$ we have $v=u \bullet w \equiv u+d h \equiv 0(\bmod L \otimes D)$. On the other hand, $e^{\chi(w)}$ belongs to the irrelevant stabilizer of $\chi\left(a_{1}\right)$ and then there exists $l \in(M \otimes A)^{-1}$ such that $e^{d k} e^{p_{1}} e^{\chi(w)}=e^{d l} e^{p_{1}}$; we can write

$$
e^{p_{2}}=e^{d k} e^{p_{1}} e^{-\chi(u)}=e^{d k} e^{p_{1}} e^{\chi(w)} e^{-\chi(w)} e^{-\chi(u)}=e^{d l} e^{p_{1}} e^{-\chi(v)} .
$$

Since $\exp \left((M \otimes D)^{0}\right)$ is a subgroup of $\exp \left((M \otimes A)^{0}\right)$ we have $e^{d l} \in \exp \left((M \otimes D)^{0}\right)$ and then $d l \in M \otimes D$. The inclusion $M \otimes D \rightarrow M \otimes A$ is a quasiisomorphism and then the cohomology class of $d l$ is trivial in $M \otimes D$. There exists $f \in(M \otimes D)^{-1}$ such that $d f=d l$ and then $e^{p_{2}}=e^{d f} e^{p_{1}} e^{-\chi(v)}$.

It is clear that every commutative diagram of morphisms of differential graded Lie algebras

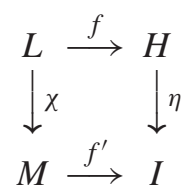

induces a natural transformation of functors

$$
\widetilde{\operatorname{Def}}_{\chi} \rightarrow \widetilde{\operatorname{Def}_{\eta}}
$$


The inverse function theorem implies that such a natural transformation is an isomorphism if and only if the pair $\left(f, f^{\prime}\right)$ induce a quasiisomorphism between the SMC of $\chi$ and $\eta$.

Example 6.13. Assume that $\chi: L \rightarrow M$ is a surjective morphism of differential graded Lie algebras, then $N=\operatorname{ker}(\chi)$ is a differential graded Lie algebra and the commutative diagram

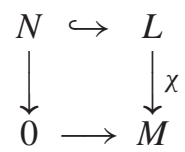

induce an isomorphism $H^{*}(N) \stackrel{\simeq}{\longrightarrow} H^{*}\left(C_{\chi}\right)$. Therefore the natural transformation $\widetilde{\operatorname{Def}}_{N} \rightarrow \widetilde{\operatorname{Def}_{\chi}}$ is an isomorphism.

The next theorem shows that, even if $\chi$ is not surjective, there exists a differential graded Lie algebra $H$ such that $\widetilde{\operatorname{Def}}_{H} \simeq \widetilde{\operatorname{Def}}_{\chi}$.

Denote by $M[t, d t]=M \otimes \mathbb{C}[t, d t]$, where $\mathbb{C}[t, d t]$ is the polynomial De Rham algebra of the affine line. More precisely $\mathbb{C}[t, d t]=\mathbb{C}[t] \oplus \mathbb{C}[t] d t, t$ has degree $0, d t$ has degree 1 and $d(p(t)+q(t) d t)=p^{\prime}(t) d t$. The inclusion $\mathbb{C} \rightarrow \mathbb{C}[t, d t]$ is a quasiisomorphism and then, by Künneth formula, also the inclusion $i: M \rightarrow M[t, d t]$ is a quasiisomorphism of differential graded Lie algebras. Define, for every $a \in \mathbb{C}$, the evaluation morphism

$$
e_{a}: M[t, d t] \rightarrow M, \quad e_{a}\left(\sum m_{i} t^{i}+n_{i} t^{i} d t\right)=\sum m_{i} a^{i},
$$

is a morphism of DGLA which is a left inverse of the inclusion $i$; in particular $e_{a}$ is a surjective quasiisomorphism for every $a$.

Theorem 6.14. For every morphism $\chi: L \rightarrow M$ of differential graded Lie algebras, the fiber product

$$
H=\left\{(l, m) \in L \times M[t, d t] \mid e_{0}(m)=0, e_{1}(m)=\chi(l)\right\}
$$

is a differential graded Lie algebra and $\widetilde{\operatorname{Def}_{H}}=\widetilde{\operatorname{Def}_{\chi}}$.

Proof. Setting

$$
K=\left\{(l, m) \in L \times M[t, d t] \mid e_{1}(m)=\chi(l)\right\}
$$

we have a commutative diagram

$$
\begin{array}{lll}
L & \stackrel{f}{\longrightarrow} & K \\
\downarrow & & \downarrow \\
M & \stackrel{I d}{\longrightarrow} & M
\end{array} \quad f(l)=(l, \chi(l)) .
$$

Passing to SMC we get an isomorphism $(f, I d): H^{*}\left(C_{\chi}\right) \rightarrow H^{*}\left(C_{e_{0}}\right)$ and $H$ is the kernel of the surjective morphism $e_{0}$. 
Proof of Theorem 2.1. By definition, for every $(A, \mathfrak{m}) \in$ Art and every $\chi: L \rightarrow M$ we have $\operatorname{Def}_{\chi}(A)=\widetilde{\operatorname{Def}}_{\chi}(\mathfrak{m})$. The proof follows from Theorem 6.12 and inverse function Theorem 6.7 .

\section{A new look at Cartan formulas}

For every differential graded vector space $\left(V, d_{V}\right)$ and every integer $i \in \mathbb{Z}$, we define the shifted differential graded vector space $\left(V[i], d_{V[i]}\right)$ by setting

$$
V[i]^{j}=V^{i+j}, \quad d_{V[i]}=(-1)^{i} d_{V} .
$$

Next, for every pair $V, W$ of differential graded vector spaces we define

$$
\operatorname{Htp}(V, W)=\operatorname{Hom}^{*}(V[1], W) .
$$

In other terms, for every integer $i$ we have

$$
\operatorname{Htp}^{i}(V, W)=\operatorname{Hom}^{i}(V[1], W)=\operatorname{Hom}^{i-1}(V, W)
$$

and the differential of $\operatorname{Htp}(V, W)$ is given by the formula

$$
\operatorname{Htp}^{i}(V, W) \ni f \mapsto \delta(f)=d_{W} f-(-1)^{i} f d_{V[1]}=d_{W} f+(-1)^{i} f d_{V} .
$$

Let $X$ be a complex manifold with Kodaira-Spencer algebra $A_{X}^{0, *}\left(T_{X}\right)$ and denote by $\left(A_{X}, d\right)$ the De Rham complex of $X$, i.e. $A_{X}=\oplus_{p, q} A^{p, q}$ and $d=\partial+\bar{\partial}$.

The contraction map is the linear map

$$
i: A_{X}^{0, *}\left(T_{X}\right) \rightarrow \operatorname{Hom}^{*}\left(A_{X}, A_{X}\right)
$$

defined as

$$
\left.\boldsymbol{i}_{a}(\omega)=a\right\lrcorner \omega, \quad a \in A_{X}^{0, *}\left(T_{X}\right), \quad \omega \in A_{X} .
$$

Note that $i: A_{X}^{0, i}\left(T_{X}\right) \rightarrow \oplus_{h, l} \operatorname{Hom}_{\mathbb{C}}\left(A_{X}^{h, l}, A_{X}^{h-1, l+i}\right) \subset \operatorname{Hom}^{i-1}\left(A_{X}, A_{X}\right)$ and then $i$ has degree -1 .

Denoting by $[$,$] the standard bracket in the DGLA Hom* \left(A_{X}, A_{X}\right)$, we have the Cartan formulas (for a proof see e.g. [27], [28])

$$
\boldsymbol{i}_{d a}=\left[\bar{\partial}, \boldsymbol{i}_{a}\right], \quad \boldsymbol{i}_{[a, b]}=\left[\boldsymbol{i}_{a},\left[\partial, \boldsymbol{i}_{b}\right]\right]=\left[\left[\boldsymbol{i}_{a}, \partial\right], \boldsymbol{i}_{b}\right], \quad\left[\boldsymbol{i}_{a}, \boldsymbol{i}_{b}\right]=0 .
$$

In order to interpret $\boldsymbol{i}$ as a morphism of differential graded Lie algebras we need to consider the DGLA given by the differential graded vector space $H \operatorname{tp}\left(\operatorname{ker}(\partial), \frac{A_{X}}{\partial A_{X}}\right)$ (whose differential is $\delta(f)=\bar{\partial} f+(-1)^{\operatorname{deg}(f)} f \bar{\partial}$ ) with the bracket

$$
\{f, g\}=f \partial g-(-1)^{\operatorname{deg}(f) \operatorname{deg}(g)} g \partial f .
$$


Proposition 7.1. The linear map

$$
i: A_{X}^{0, *}\left(T_{X}\right) \rightarrow \operatorname{Htp}\left(\operatorname{ker}(\partial), \frac{A_{X}}{\partial A_{X}}\right)
$$

is a morphism of differential graded Lie algebras.

Proof. Straightforward consequence of Cartan formulas.

Consider now a smooth closed submanifold $Z \subset X$ and denote by $I_{Z} \subset A_{X}$ the graded subspace of differential forms vanishing on $Z$. Since

$$
A_{X}^{0, *}\left(T_{X}\right)(-\log Z) \subset\left\{a \in A_{X}^{0, *}\left(T_{X}\right) \mid i_{a}\left(I_{Z}\right) \subset I_{Z}\right\},
$$

we have a commutative diagram of morphisms of DGLA

$$
\begin{aligned}
& A_{X}^{0, *}\left(T_{X}\right)(-\log Z) \stackrel{i}{\longrightarrow}\left\{f \in \operatorname{Htp}\left(\operatorname{ker}(\partial), \frac{A_{X}}{\partial A_{X}}\right) \mid f\left(I_{Z} \cap \operatorname{ker}(\partial)\right) \subset \frac{I_{Z}}{I_{Z} \cap \partial A_{X}}\right\} \\
& \downarrow x \downarrow \eta \\
& A_{X}^{0, *}\left(T_{X}\right) \quad \stackrel{i}{\longrightarrow} \operatorname{Htp}\left(\operatorname{ker}(\partial), \frac{A_{X}}{\partial A_{X}}\right) \text {. }
\end{aligned}
$$

Notice that

$$
\operatorname{Coker}(\eta)=\operatorname{Htp}\left(I_{Z} \cap \operatorname{ker}(\partial), \frac{A_{Z}}{\partial A_{Z}}\right) .
$$

Lemma 7.2. If the differential graded vector spaces $\left(\partial A_{X}, \bar{\partial}\right)$ and $\left(\partial A_{Z}, \bar{\partial}\right)$ are acyclic, then the functor $\operatorname{Def}_{\eta}$ is unobstructed. In particular the obstructions of $\operatorname{Def}_{\chi}=\operatorname{Hilb}_{X}^{Z}$ are contained in the kernel of

$$
H^{2}\left(C_{\chi}\right) \stackrel{i}{\longrightarrow} H^{2}\left(C_{\eta}\right)
$$

(Notice that $H^{2}\left(C_{\chi}\right)=H^{1}\left(Z, N_{Z \mid X}\right)$ and

$$
H^{2}\left(C_{\eta}\right)=H^{1}(\operatorname{Coker}(\eta))=\oplus_{i} \operatorname{Hom}\left(H^{i}\left(I_{Z} \cap \operatorname{ker}(\partial)\right), H^{i}\left(\frac{A_{Z}}{\partial A_{Z}}\right)\right) .
$$

Proof. We first note that the exact sequence

$$
0 \longrightarrow I_{Z} \cap \partial A_{X} \longrightarrow \partial A_{X} \longrightarrow \partial A_{Z} \longrightarrow 0
$$

implies that also the complex $I_{Z} \cap \partial A_{X}$ is acyclic. For simplicity of notation denote

$$
K=\left\{f \in \operatorname{Htp}\left(\operatorname{ker}(\partial), \frac{A_{X}}{\partial A_{X}}\right) \mid f\left(I_{Z} \cap \operatorname{ker}(\partial)\right) \subset \frac{I_{Z}}{I_{Z} \cap \partial A_{X}}\right\} .
$$


The projection $\operatorname{ker}(\partial) \rightarrow \operatorname{ker}(\partial) / \partial A_{X}$ induces a commutative diagram

$$
\begin{array}{ccc}
\left\{f \in K \mid f\left(\partial A_{X}\right)=0\right\} & \stackrel{\alpha}{\longrightarrow} & K \\
\downarrow \mu & \downarrow \eta \\
\operatorname{Htp}\left(\frac{\operatorname{ker}(\partial)}{\partial A_{X}}, \frac{A_{X}}{\partial A_{X}}\right) & \stackrel{\beta}{\longrightarrow} \operatorname{Htp}\left(\operatorname{ker}(\partial), \frac{A_{X}}{\partial A_{X}}\right) .
\end{array}
$$

Since $\partial A_{X}$ is acyclic, $\beta$ is a quasiisomorphism of DGLA.

Since

$$
\operatorname{Coker}(\alpha)=\left\{f \in \operatorname{Htp}\left(\partial A_{X}, \frac{A_{X}}{\partial A_{X}}\right) \mid f\left(I_{Z} \cap \partial A_{X}\right) \subset \frac{I_{Z}}{I_{Z} \cap \partial A_{X}}\right\} .
$$

there exists an exact sequence

$$
0 \rightarrow \operatorname{Htp}\left(\frac{\partial A_{X}}{I_{Z} \cap \partial A_{X}}, \frac{A_{X}}{\partial A_{X}}\right) \rightarrow \operatorname{Coker} \alpha \rightarrow \operatorname{Htp}\left(I_{Z} \cap \partial A_{X}, \frac{I_{Z}}{I_{Z} \cap \partial A_{X}}\right) \rightarrow 0 .
$$

Since the complexes $\frac{\partial A_{X}}{I_{Z} \cap \partial A_{X}}=\partial A_{Z}$ and $I_{Z} \cap \partial A_{X}$ are both acyclic, also $\operatorname{Coker}(\alpha)$ is acyclic and then $\alpha$ is a quasiisomorphism. According to Theorem 2.1 there exists an isomorphism of functors $\operatorname{Def}_{\eta}=\operatorname{Def}_{\mu}$. On the other side, both algebras on the first column are Abelian and then, by Lemma 2.2 the functor $\operatorname{Def}_{\mu}$ is smooth. The vanishing of obstructions follows from Proposition 2.3.

\section{Examples and applications}

In the notation of previous section, the contraction operator gives a morphism of complexes

$$
\boldsymbol{i}: A_{X}^{0, *}\left(T_{X}\right) \rightarrow \mathrm{Htp}\left(\operatorname{ker}(\partial), A_{X}\right),
$$

where the differential on $\operatorname{Htp}\left(\operatorname{ker}(\partial), A_{X}\right)$ is $f \mapsto \bar{\partial} f \pm f \bar{\partial}$. The inclusion $I_{Z} \cap$ $\operatorname{ker}(\partial) \subset \operatorname{ker}(\partial)$ and the projection $A_{X} \rightarrow A_{Z}$ give a morphism of complexes

$$
\pi_{Z}: \operatorname{Htp}\left(\operatorname{ker}(\partial), A_{X}\right) \rightarrow \operatorname{Htp}\left(I_{Z} \cap \operatorname{ker}(\partial), A_{Z}\right) .
$$

The subalgebra $A_{X}^{0, *}\left(T_{X}\right)(-\log Z)$ is contained in the kernel of the composition and therefore there exists a quotient map

$$
\pi_{Z} \boldsymbol{i}: A_{Z}^{0, *}\left(N_{Z \mid X}\right) \rightarrow \operatorname{Htp}\left(I_{Z} \cap \operatorname{ker}(\partial), A_{Z}\right) .
$$

If $\omega \in I_{Z}$ is a closed $(p, q)$-form, then $\partial \omega=\bar{\partial} \omega=0$ and then gives a morphism of complexes

$$
\lrcorner \omega:\left(A_{Z}^{0, *}\left(N_{Z \mid X}\right), \bar{\partial}\right) \rightarrow\left(A_{Z}^{p-1, q+*}, \bar{\partial}\right), \quad \eta\right\lrcorner \omega=\pi_{Z} \boldsymbol{i}_{\eta}(\omega) .
$$


Therefore Theorem 0.1 is completely equivalent to

Theorem 8.1. In the above notation, if $X$ is compact Kähler, then the obstruction to $\mathrm{Hilb}_{X}^{Z}$ are contained in the kernel of

$$
\pi_{Z} \boldsymbol{i}: H^{1}\left(A_{Z}^{0, *}\left(N_{Z \mid X}\right)\right) \rightarrow H^{1}\left(\operatorname{Htp}\left(I_{Z} \cap \operatorname{ker}(\partial), A_{Z}\right)\right)
$$

Proof. Since $X$ is compact Kähler, the subcomplex $\operatorname{Im}(\partial)=\partial A_{X} \subset A_{X}$ is acyclic. In fact by $\partial \bar{\partial}$-lemma we have

$$
\operatorname{ker}(\bar{\partial}) \cap \operatorname{Im}(\partial)=\operatorname{ker}(\partial) \cap \operatorname{Im}(\bar{\partial})=\operatorname{Im}(\partial \bar{\partial})
$$

and the equality $\operatorname{ker}(\bar{\partial}) \cap \operatorname{Im}(\partial)=\operatorname{Im}(\partial \bar{\partial})$ implies immediately that $H_{\bar{\partial}}^{*}(\operatorname{Im}(\partial))=0$. Moreover, also $Z$ is Kähler and then the same conclusion holds for the complex $\partial A_{Z}$. In particular the projection

$$
\operatorname{Htp}\left(I_{Z} \cap \operatorname{ker}(\partial), A_{Z}\right) \rightarrow \operatorname{Htp}\left(I_{Z} \cap \operatorname{ker}(\partial), \frac{A_{Z}}{\partial A_{Z}}\right)
$$

is a quasiisomorphism. By Lemma 7.2, the obstruction space of $\mathrm{Hilb}_{X}^{Z}$ is contained in the kernel of the linear map

$$
\begin{aligned}
H^{1}\left(N_{Z \mid X}\right) \stackrel{i}{\longrightarrow} & H^{1}\left(\operatorname{Htp}\left(I_{Z} \cap \operatorname{ker}(\partial), \frac{A_{Z}}{\partial A_{Z}}\right)\right) \\
& \bigoplus_{i} \operatorname{Hom}\left(H^{i}\left(I_{Z} \cap \operatorname{ker}(\partial)\right), H^{i}\left(\frac{A_{Z}}{\partial A_{Z}}\right)\right) .
\end{aligned}
$$

The interplay between semiregularity and embedded deformations has been studied by Severi [33] for curves on surfaces, by Kodaira and Spencer [22] for submanifolds of codimension 1 and by S. Bloch [3] for every submanifolds of a projective variety. They proved that if the semiregularity map is injective, then the corresponding embedded deformations are unobstructed.

Corollary 8.2. Let $Z$ be a smooth closed submanifold of codimension p of a compact Kähler manifold $X$. Then the obstruction space of $\mathrm{Hilb}_{X}^{Z}$ is contained in the kernel of the semiregularity map

$$
H^{1}\left(Z, N_{Z \mid X}\right) \rightarrow H^{p+1}\left(X, \Omega_{X}^{p-1}\right) .
$$

Proof. Under our assumption the semiregularity map can be defined in the following way: let $n$ be the dimension of $X$ and denote by $\mathcal{H}$ the space of harmonic forms on $X$ of type $(n-p+1, n-p-1)$. By Dolbeault theorem and Serre duality, the 
dual of $\mathcal{H}$ is isomorphic to $H^{p+1}\left(X, \Omega_{X}^{p-1}\right)$. The composition of the contraction map and integration on $Z$ gives a bilinear map

$$
\left.H^{1}\left(Z, N_{Z \mid X}\right) \times \mathcal{H} \rightarrow H^{n-p}\left(Z, \Omega_{Z}^{n-p}\right) \rightarrow \mathbb{C}, \quad(\eta, \omega)=\int_{Z} \eta\right\lrcorner \omega
$$

which induces the semiregularity map

$$
H^{1}\left(Z, N_{Z \mid X}\right) \rightarrow \mathcal{H}^{\vee}=H^{p+1}\left(X, \Omega_{X}^{p-1}\right)
$$

Since $\mathcal{H} \subset I_{Z} \cap \operatorname{ker}(\partial) \cap \operatorname{ker}(\bar{\partial})$, the proof follows immediately from Theorem 0.1 .

Remark 8.3. It is not clear to me if Corollary 8.2 is valid without the Kähler assumption; a closer look to the proofs shows that the $\partial \bar{\partial}$-lemma on $Z$ is not required and then the Kähler assumption can be weakened to the validity of the $\partial \bar{\partial}$-lemma on $X$. Therefore, according to [7, Corollary 5.23], the Corollary 8.2 holds for every compact complex manifold $X$ which can be blown up to a Kähler manifold; in particular it holds for every Moishezon variety.

Remark 8.4. Corollary 8.2 was almost proved by S. Bloch in the paper [3]. More precisely he proved that if the semiregularity map is injective then $\mathrm{Hilb}_{X}^{Z}$ is smooth; although not explicitly stated in [3], the same proof shows that the semiregularity map annihilates obstructions arising from small extensions

$$
0 \longrightarrow J \stackrel{f}{\longrightarrow} A \longrightarrow B \longrightarrow 0
$$

of Artin rings where the differential $d f: J \rightarrow \Omega_{A} \otimes_{A} B$ is injective.

Corollary 8.2 appears also in [30] with an incorrect proof, as pointed out by Kawamata in [20]. However [30] still contains a clever and simple proof of Bloch's theorem.

Corollary 8.5 (Voisin [35]). Let $X$ be a Kähler and holomorphic symplectic variety and let $Z \subset X$ be a holomorphic lagrangian submanifold. Then $\mathrm{Hilb}_{X}^{Z}$ is smooth.

Proof. Let $\omega$ be the holomorphic symplectic $(2,0)$-form on $X$. If $Z$ is a lagrangian submanifold then $\omega_{\mid Z}=0$ and the contraction with $\omega$ gives an isomorphism of vector bundles over $Z$

$$
\lrcorner \omega: N_{Z \mid X} \stackrel{\simeq}{\longrightarrow} \Omega_{Z}^{1}
$$

In particular the map

$$
\lrcorner \omega: H^{1}\left(Z, N_{Z \mid X}\right) \rightarrow H^{1}\left(Z, \Omega_{Z}^{1}\right)
$$

is injective and then, according to Theorem 0.1 , every obstruction of $\operatorname{Hilb}_{X}^{Z}$ vanishes. 


\section{References}

[1] M. ARTin, "Deformations of Singularities", Tata Institute of Fundamental Research, Bombay, 1976.

[2] K. Behrend and B. FAntechi, The intrinsic normal cone, Invent. Math. 128 (1997), $45-88$.

[3] S. BLOCH, Semi-regularity and de Rham cohomology, Invent. Math. 17 (1972) 51-66.

[4] R. O. BUCHWEITZ and H. FLENNER, A semiregularity map for modules and applications to deformations, Compositio Math. 137 (2003), 135-210. arXiv: math. AG / 9912245

[5] P. BURCHARD and H. Clemens, Normal differential operators and deformation theory, In: "Recent progress in intersection theory" (Bologna, 1997), Birkhäuser Boston, 2000, 33-84. arXiv:math. AG/9811171

[6] H. Clemens, Geometry of formal Kuranishi theory. Adv. Math. 198 (2005), 311-365.

[7] P. Deligne, P. Griffiths, J. Morgan and D. Sullivan, Real homotopy theory of Kähler manifolds, Invent. Math. 29 (1975), 245-274.

[8] S. K. Donaldson and P. B. Kronheimer, "The Geometry of Four-Manifolds", Oxford, University Press, 1990.

[9] A. DouADY, Obstruction primaire à la déformation, Sém. Cartan 13 (1960/61), Exp. 4.

[10] B. Fantechi and M. ManetTi, Obstruction calculus for functors of Artin rings, I. J. Algebra 202 (1998), 541-576.

[11] D. Fiorenza and M. MANETTI, $L_{\infty}$ structures on mapping cones, Algebra Number Theory 1 (2007), 301-330.

[12] K. FuKaYA, Deformation theory, homological algebra and mirror symmetry, In: "Geometry and Physics of Branes" (Como, 2001), Ser. High Energy Phys. Cosmol. Gravit., IOP Bristol, 2003, 121-209.

[13] W. M. Goldman and J. J. Millson, The deformation theory of representations of fundamental groups of compact Kähler manifolds, Inst. Hautes Études Sci. Publ. Math. 67 (1988), 43-96.

[14] W. M. Goldman and J. J. Millson, The homotopy invariance of the Kuranishi space, Illinois J. Math. 34 (1990), 337-367.

[15] M. Green and R. LAZARSFElD, Deformation theory, generic vanishing theorems, and some conjectures of Enriques, Catanese and Beauville, Invent. Math. 90 (1987), 389-407.

[16] M. GREEN and R. LAZARSFELD, Higher obstructions to deforming cohomology groups of line bundles, J. Amer. Math. Soc. 4 (1991), 87-103.

[17] R. HARTShORne, "Residues and Duality", Springer-Verlag, L.N.M., Vol. 20, 1966.

[18] D. IACONO, "Differential Graded Lie Algebras and Deformations of Holomorphic Maps", $\mathrm{PhD}$ thesis, 2006, arXiv:math. AG/ 0701091

[19] N. JACOBSON, "Lie Algebras", Wiley \& Sons, 1962.

[20] Y. KAWAMATA, Unobstructed deformations - a remark on a paper of Z. Ran, J. Algebraic Geom. 1 (1992), 183-190.

[21] S. Kobayashi, "Differential Geometry of Complex Vector Bundles", Princeton, Univ. Press, 1987.

[22] K. KodAira and D. C. SPENCER, A theorem of completeness of characteristic systems of complete continuous systems, Amer. J. Math. 81 (1959), 477-500.

[23] J. KolláR, "Rational curves on algebraic varieties", Springer-Verlag, Ergebnisse Vol. 32, 1996.

[24] M. Kontsevich, Deformation quantization of Poisson manifolds, I, Lett. Math. Phys. 66 (2003), 157-216. arXiv: q-alg/9709040

[25] M. ManetTi, Deformation theory via differential graded Lie algebras, In: "Seminari di Geometria Algebrica 1998-1999”, Scuola Normale Superiore, 1999.

arXiv:math.AG/0507284

[26] M. ManetTI, Extended deformation functors, Int. Math. Res. Not. 14 (2002), 719-756. arXiv:math.AG/9910071 
[27] M. ManetTi, Cohomological constraint to deformations of compact Kähler manifolds, Adv. Math. 186 (2004), 125-142.

[28] M. MAnetTi, Lectures on deformations on complex manifolds, Rend. Mat. Appl. 24 (2004), 1-183. arXiv: math. AG/0507286

[29] V. P. Palamodov, Deformations of complex spaces, Uspekhi Mat. Nauk. 31:3 (1976), 129-194. Transl. Russian Math. Surveys 31:3 (1976), 129-197.

[30] Z. RAN, Hodge theory and the Hilbert scheme, J. Differential Geom. 37 (1993), 191-198.

[31] M. SCHLESSINGER, Functors of Artin rings, Trans. Amer. Math. Soc. 130 (1968), 208-222.

[32] M. Schlessinger and J. StASHefF, Deformation Theory and Rational Homotopy Type, preprint, 1979.

[33] F. SEVERI, Sul teorema fondamentale dei sistemi continui di curve sopra una superficie algebrica, Ann. Mat. Pura Appl. 23 (1944), 149-181.

[34] D. TANRÉ, "Homotopie Rationelle: Modèles de Chen, Quillen, Sullivan", Springer-Verlag, Lecture Notes in Mathematics Vol. 1025, 1983.

[35] C. Voisin, Sur la stabilité de sous-variétés lagrangiennes des variétés symplectiques holomorphes, In: "Complex Projective Geometry" (Trieste, 1989/Bergen, 1989), London Math. Soc., Lecture Note Ser. Vol. 179, 1992, 294-303.

Dipartimento di Matematica "Guido Castelnuovo" Sapienza Università di Roma

P.le Aldo Moro 5

I-00185 Roma, Italy

manetti@mat.uniroma1.it 